\title{
Companions on Artin stacks
}

\author{
Weizhe Zheng*广
}

\begin{abstract}
Deligne's conjecture that $\ell$-adic sheaves on normal schemes over a finite field admit $\ell^{\prime}$-companions was proved by L. Lafforgue in the case of curves and by Drinfeld in the case of smooth schemes. In this paper, we extend Drinfeld's theorem to smooth Artin stacks and deduce Deligne's conjecture for coarse moduli spaces of smooth Artin stacks. We also extend related theorems on Frobenius eigenvalues and traces to Artin stacks.
\end{abstract}

\section{Introduction}

Let $\mathbb{F}_{q}$ be a finite field and let $\ell$ and $\ell^{\prime}$ be prime numbers not dividing $q$. We let $\overline{\mathbb{Q}_{\ell}}$ denote an algebraic closure of $\mathbb{Q}_{\ell}$. Deligne conjectured [D2, Conjecture 1.2.10] that every lisse

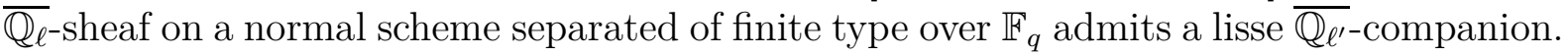
Drinfeld [D4, Theorem 1.1] proved this conjecture for smooth schemes. The goal of this paper is to extend Drinfeld's theorem to smooth Artin stacks. We deduce that Deligne's conjecture holds for coarse moduli spaces of smooth Artin stacks. We also extend related theorems on Frobenius eigenvalues and traces to Artin stacks.

For an Artin stack $X$ of finite presentation over $\mathbb{F}_{q}$ and a Weil $\overline{\mathbb{Q}_{\ell}}$-sheaf $\mathcal{F}$ on $X$, we let $E(\mathcal{F})$ denote the subfield of $\overline{\mathbb{Q}_{\ell}}$ generated by the local Frobenius traces $\operatorname{tr}\left(\operatorname{Frob}_{x}, \mathcal{F}_{\bar{x}}\right)$, where $x \in X\left(\mathbb{F}_{q^{n}}\right)$ and $n \geq 1$. Here Frob $_{x}=$ Frob $_{q^{n}}$ denotes the geometric Frobenius, and $\bar{x}$ denotes a geometric point above $x$. Let $E_{\lambda^{\prime}}$ be an algebraic extension of $\mathbb{Q}_{\ell^{\prime}}$ and let $\sigma: E(\mathcal{F}) \rightarrow E_{\lambda^{\prime}}$ be a field embedding, not necessarily continuous. We say that a

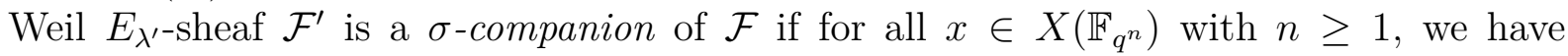
$\operatorname{tr}\left(\operatorname{Frob}_{x}, \mathcal{F}_{\bar{x}}^{\prime}\right)=\sigma \operatorname{tr}\left(\operatorname{Frob}_{x}, \mathcal{F}_{\bar{x}}\right)$.

Our main results on Frobenius eigenvalues and traces are as follows.

Theorem 0.1. Let $X$ be a geometrically unibranch ${ }^{1}$ Artin stack of finite presentation over $\mathbb{F}_{q}$ and let $\mathcal{F}$ be a simple lisse $\overline{\mathbb{Q}_{\ell}}$-sheaf of rank $r$ on $X$ such that $\operatorname{det}(\mathcal{F})$ has finite order.

*Morningside Center of Mathematics and Hua Loo-Keng Key Laboratory of Mathematics, Academy of Mathematics and Systems Science, Chinese Academy of Sciences, Beijing 100190, China; University of the Chinese Academy of Sciences, Beijing 100049, China; email: wzheng@math.ac.cn. Partially supported by China's Recruitment Program of Global Experts; National Natural Science Foundation of China Grants 11321101, 11621061, 11688101; National Center for Mathematics and Interdisciplinary Sciences, Chinese Academy of Sciences.

†Mathematics Subject Classification 2010: 14F20 (Primary); 14G15, 14A20, 14D22 (Secondary).

${ }^{1}$ For a short review of the property "geometrically unibranch", see Remark 1.5 . 
(1) (Frobenius eigenvalues) Let $x \in X\left(\mathbb{F}_{q^{n}}\right)$ and let $\alpha$ be an eigenvalue of Frob acting $_{x}$ on $\mathcal{F}_{\bar{x}}$. Then $\alpha$ is a $q$-Weil number of weight 02 Moreover, for every valuation $v$ on $\mathbb{Q}(\alpha)$ such that $v\left(q^{n}\right)=1$, we have $|v(\alpha)| \leq \frac{r-1}{2}$.

(2) (Frobenius traces) The field $E(\mathcal{F})$ is a number field (namely, a finite extension of $\mathbb{Q})$.

The statement of Theorem 0.1, with a slightly weaker bound for the $p$-adic valuations, is conjectured to hold for normal schemes separated of finite type over $\mathbb{F}_{q}$ by Deligne [D2, Conjecture 1.2.10 (i)-(iv)]. In the case of curves, the theorem with a weaker bound is a consequence of the Langlands correspondence for $\mathrm{GL}(n)$ over function fields proved by L. Lafforgue [L1, Théorème VII.6]. The improvement of the bound is due to V. Lafforgue [L1, Corollaire 2.2]. The extension from curves to schemes is stated by L. Lafforgue [L1, Proposition VII.7] for part (1), and due to Deligne [D3, Théorème 3.1] for part (2).

Recently Drinfeld and Kedlaya [DK, Theorem 1.3.3] proved a refinement of V. Lafforgue's bound for Newton polygons, which can be thought of as an analogue of Griffiths transversality. We also extend this result to stacks (Theorem 2.6).

The following is our main result on companions.

Theorem 0.2 (Companions on smooth stacks). Let $X$ be a smooth Artin stack over $\mathbb{F}_{q}$ of finite presentation and separated diagonal. Let $\mathcal{F}$ be a lisse Weil $\overline{\mathbb{Q}_{\ell}}$-sheaf on $X$. Then, for every embedding $\sigma: E(\mathcal{F}) \rightarrow \overline{\mathbb{Q}_{\ell^{\prime}}}, \mathcal{F}$ admits a lisse $\sigma$-companion $\mathcal{F}^{\prime}$. Moreover, if $E(\mathcal{F})$ is a number field, then there exists a finite extension $E$ of $E(\mathcal{F})$ such that for every finite place $\lambda^{\prime}$ of $E$ not dividing $q, \mathcal{F}$ admits a lisse $\sigma_{\lambda^{\prime}}$-companion. Here $\sigma_{\lambda^{\prime}}: E(\mathcal{F}) \rightarrow E \rightarrow E_{\lambda^{\prime}}$, and $E_{\lambda^{\prime}}$ denotes the completion of $E$ at $\lambda^{\prime}$.

The statements of Theorem 0.2 are conjectured to hold for normal schemes separated of finite type over $\mathbb{F}_{q}$ by Deligne [D2, Conjecture 1.2.10 (v)]. In the case of curves, the first assertion of the theorem is due to L. Lafforgue [L1, Théorème VII.6], and the second to Chin [C]. The extension from curves to smooth schemes is due to Drinfeld [D4, Theorem $1.1]$.

As an application of Theorem 0.2 , we deduce that Deligne's conjecture holds for coarse moduli spaces of smooth Artin stacks.

Corollary 0.3 (Companions on coarse moduli spaces). Let $X$ be a scheme or algebraic space that is Zariski locally the coarse moduli space of a smooth Artin stack of finite inertia and finite presentation over $\mathbb{F}_{q}$ (e.g. when $X$ has quotient singularities). Then the statements of Theorem 0.2 hold for $X$.

The general normal case seems difficult. Drinfeld deduces his result from an equivalence between lisse sheaves on a regular scheme $X$ and compatible systems of lisse sheaves on curves on $X$ [D4, Theorem 2.5]. This equivalence fails for $X$ normal in general [D4, Section 6].

For both Theorems 0.1 and 0.2 , we reduce first to the case of a quotient stack $[Y / G]$ of a quasi-projective scheme $Y$ by a finite group $G$, and then, choosing an embedding $G \rightarrow \mathrm{GL}_{m}$, to the scheme $Y \wedge^{G} \mathrm{GL}_{m}$. One step of the reduction consists of showing that the assertions can be checked on any dense open substack.

\footnotetext{
${ }^{2}$ We adopt the convention that a $q$-Weil number of weight 0 is an algebraic number $\alpha$ such that for every place $\lambda$ of $\mathbb{Q}(\alpha)$ not dividing $q$ (finite or Archimedean), we have $|\alpha|_{\lambda}=1$.
} 
Gabber's theorem [F] that companionship is preserved by operations on the Grothendieck groups extends to stacks [Z3, Proposition 5.8]. Combining this with Theorem 0.2, one obtains the existence of perverse companions on (not necessarily smooth) Artin stacks.

The paper is organized as follows. In Section 1, we establish some preliminary results on Weil sheaves. In Section 2, we prove Theorems 0.1 (1) on Frobenius eigenvalues and Theorem 2.6 on Newton polygons. We deduce from Theorem 0.1 (1) that the bounded derived category $D_{c}^{b}\left(X, \overline{\mathbb{Q}_{\ell}}\right)$ is a direct sum of twists of the derived category of weakly motivic complexes for any Artin stack $X$ of finite presentation over $\mathbb{F}_{q}$. In Section 3 , we prove Theorem 0.1 (2) on Frobenius traces. In Section 4, we prove Theorem 0.2 and Corollary 0.3 on lisse companions. We deduce results on perverse companions and companions in Grothendieck groups on Artin stacks of finite presentation and separated diagonal over $\mathbb{F}_{q}$. In an appendix (Section [5), we prove that pure perverse sheaves on $X$ are geometrically semisimple, without assuming that the stabilizers are affine, extending a result of Sun [S2, Theorem 3.11].

Unless otherwise stated, all stacks are assumed to be Artin stacks of finite presentation over $\mathbb{F}_{q}$, not necessarily of separated diagonal, and sheaves are assumed to be constructible. We write $D\left(X, \overline{\mathbb{Q}_{\ell}}\right)$ for $D_{c}\left(X, \overline{\mathbb{Q}_{\ell}}\right)$. We will only consider the middle perversity.

\section{Acknowledgments}

This paper grows out of an answer to Shenghao Sun's question of extending the theorems of Deligne and Drinfeld to stacks. I thank Yongquan Hu, Yifeng Liu, Martin Olsson, and Shenghao Sun for useful discussions, and Vladimir Drinfeld and Luc Illusie for valuable comments. I am grateful to Ofer Gabber for pointing out a mistake in a draft of this paper. I thank the referee for a careful reading of the manuscript and many helpful comments. Part of this paper was written during a stay at Shanghai Center for Mathematical Sciences and I thank the center for hospitality.

\section{Weil sheaves}

For problems concerning companions, it is convenient to work with Weil sheaves. In this section, we establish some preliminary results on Weil sheaves. The main result is Proposition 1.8 on the determinant of lisse Weil $\overline{\mathbb{Q}_{\ell}}$-sheaves on geometrically unibranch stacks. We deduce that the category of Weil $\overline{\mathbb{Q}}{ }_{\ell}$-sheaves is a direct sum of the twists of the category of $\overline{\mathbb{Q}_{\ell}}$-sheaves (Proposition 1.15 ).

Let $E_{\lambda}$ be an algebraic extension of $\mathbb{Q}_{\ell}$. A Weil $E_{\lambda}$-sheaf on a stack $X$ is an $E_{\lambda}$-sheaf $\mathcal{F}$ on $X \otimes_{\mathbb{F}_{q}} \overline{\mathbb{F}_{q}}$ equipped with an action of the Weil group $W\left(\overline{\mathbb{F}_{q}} / \mathbb{F}_{q}\right)$ lifting the action

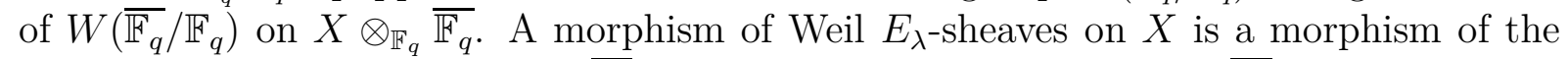
underlying $E_{\lambda}$-sheaves on $X \otimes_{\mathbb{F}_{q}} \overline{\mathbb{F}_{q}}$ compatible with the action of $W\left(\overline{\mathbb{F}_{q}} / \mathbb{F}_{q}\right)$.

Remark 1.1. More formally, the category $\operatorname{Shv}^{W}\left(X, E_{\lambda}\right)$ of Weil $E_{\lambda}$-sheaves on $X$ is a (pseudo)limit of the diagram (i.e. pseudofunctor) $B \mathbb{Z} \rightarrow \mathcal{C}$ at given by the action of $\mathbb{Z} \simeq W\left(\overline{\mathbb{F}_{q}} / \mathbb{F}_{q}\right)$ on the category $\operatorname{Shv}\left(X \otimes_{\mathbb{F}_{q}} \overline{\mathbb{F}_{q}}, E_{\lambda}\right)$, where $B \mathbb{Z}$ is the groupoid associated to the group $\mathbb{Z}$ and $\mathcal{C}$ at is the 2-category of categories. If we let $\mathcal{C} a t^{B \mathbb{Z}}$ denote the 2category of diagrams $B \mathbb{Z} \rightarrow \mathcal{C}$ at, the forgetful 2-functor for $: \mathcal{C} a t^{B \mathbb{Z}} \rightarrow \mathcal{C}$ at and the limit

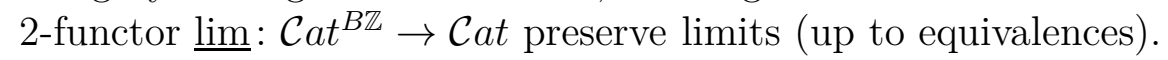

Remark 1.2. The functor $\operatorname{Shv}\left(X, E_{\lambda}\right) \rightarrow \operatorname{Shv}^{W}\left(X, E_{\lambda}\right)$ carrying $\mathcal{F}$ to $\left(\mathcal{F}_{\overline{\mathbb{F}_{q}}}, \phi\right)$, where $\mathcal{F}_{\overline{\mathbb{F}_{q}}}$ is the pullback of $\mathcal{F}$ to $X \otimes_{\mathbb{F}_{q}} \overline{\mathbb{F}_{q}}$ and $\phi$ is the restriction of the action of $\operatorname{Gal}\left(\overline{\mathbb{F}_{q}} / \mathbb{F}_{q}\right)$ to 
$W\left(\overline{\mathbb{F}_{q}} / \mathbb{F}_{q}\right)$, is fully faithful. Moreover, its essential image is stable under extension by the following general facts on extensions (cf. [BBD, Proposition 5.1.2]).

(1) Let $(\mathcal{A}, F)$ be an Abelian category with $\mathbb{Z}$-action (i.e. a pseudofunctor from $B \mathbb{Z}$ to the 2-category of Abelian categories). For objects $(A, \phi)$ and $(B, \psi)$ of the limit category $\mathcal{A}^{F}$, we have a short exact sequence of Abelian groups (cf. [BBD, page 124])

$$
0 \rightarrow \operatorname{Hom}_{\mathcal{A}}(A, B)_{\mathbb{Z}} \rightarrow \operatorname{Ext}_{\mathcal{A}^{F}}^{1}((A, \phi),(B, \psi)) \rightarrow \operatorname{Ext}_{\mathcal{A}}^{1}(A, B)^{\mathbb{Z}} \rightarrow 0
$$

(2) Let $\mathcal{D}$ be a triangulated category equipped with a $t$-structure. Note that $\mathcal{D}$ is not necessarily equivalent to the derived category of its heart $\mathcal{A}$. Nonetheless, for $A$ and $B$ in $\mathcal{A}$, we have an isomorphism $\operatorname{Hom}_{\mathcal{D}}(A, B[1]) \simeq \operatorname{Ext}_{\mathcal{A}}^{1}(A, B)$ carrying $f$ to the extension given by completing $f$ into a distinguished triangle (cf. [BBD, Remarque 3.1 .17 (ii)]).

Recall from [SP, Tag 06U6] that a morphism $f: X \rightarrow Y$ of Artin stacks (not necessarily of finite presentation over $\mathbb{F}_{q}$ ) is said to be submersive if the induced continuous map is a quotient map, and universally submersive if for every morphism of Artin stacks $Y^{\prime} \rightarrow Y$, the base change $X \times_{Y} Y^{\prime} \rightarrow Y^{\prime}$ of $f$ is submersive.

Lemma 1.3. Let $f: X \rightarrow Y$ be a universally submersive morphism of stacks. Then $f$ is of effective descent for Weil $E_{\lambda}$-sheaves and for $E_{\lambda}$-sheaves.

The statement of the lemma means that $f^{*}$ induces an equivalence of categories from $\operatorname{Shv}^{W}\left(Y, E_{\lambda}\right)$ to the category of descent data, which is a limit of the diagram

$$
\operatorname{Shv}^{W}\left(X, E_{\lambda}\right) \Longrightarrow \operatorname{Shv}^{W}\left(X \times_{Y} X, E_{\lambda}\right) \Longrightarrow \operatorname{Shv}^{W}\left(X \times_{Y} X \times_{Y} X, E_{\lambda}\right)
$$

induced by inverse image functors. An object of the category of descent data is a Weil $E_{\lambda^{-}}$ sheaf $\mathcal{F}$ on $X$ endowed with an isomorphism $p_{1}^{*} \mathcal{F} \simeq p_{2}^{*} \mathcal{F}$ satisfying the cocycle condition. Here $p_{1}, p_{2}: X \times_{Y} X \rightarrow X$ are the two projections. Compare with [IZ, Proposition 2.4].

Proof. Consider the pseudofunctor $F:$ Stk $\rightarrow \mathcal{C} a t^{B \mathbb{Z}}$ carrying $X$ to the diagram in Remark 1.1, where Stk is the 2-category of stacks. The assertion of the lemma for Weil $E_{\lambda}$-sheaves is that $f$ is of effective descent for the pseudofunctor $\underline{\lim } \circ F$. Since $\underline{\text { lim }}$ and $\underline{\text { for }}$ preserve limits, we may replace $\underline{\lim } \circ F$ by $F$, and then by for $\circ F$. In other words, it suffices to show that $f \otimes_{\mathbb{F}_{q}} \overline{\mathbb{F}_{q}}$ is of effective descent for $E_{\lambda}$-sheaves.

Changing notation, let us show that any universally submersive morphism $r: X \rightarrow Y$ of Artin stacks of finite presentation over $\mathbb{F}_{q}$ or $\overline{\mathbb{F}_{q}}$ is of effective descent for $E_{\lambda^{-}}$-sheaves. Let $f: Y^{\prime} \rightarrow Y$ be a smooth presentation with $Y^{\prime}$ a scheme and let $X^{\prime} \rightarrow X \times_{Y} Y^{\prime}$ be a smooth presentation with $X^{\prime}$ a scheme. Consider the square

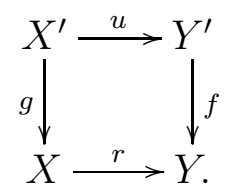

By a general property of descent [G, Théorème 10.4, line 6 of the table], it suffices to show that $u, f, g$, and $g^{\prime}: X^{\prime} \times_{Y^{\prime}} X^{\prime} \rightarrow X \times_{Y} X$ are of effective descent. Note that $f, g$, and $g^{\prime}$ are representable and smooth surjective, and $u$ is a universally submersive 
morphism of schemes. By a theorem of Voevodsky [V, Theorem 3.1.9], $u$ is dominated by $\amalg V_{i} \rightarrow V \stackrel{v}{\rightarrow} Y^{\prime}$, where $v$ is a proper surjective morphism of schemes and $\left(V_{i}\right)$ is a finite Zariski open cover of $V$. By general properties of descent [G, Proposition 6.25 (ii), (iii)], we are reduced to showing that $r$ is of effective descent in the following cases: (a) $r$ is representable and smooth surjective; (b) $r$ is a proper surjective morphism of schemes. This follows from Beck's theorem [BR, Proposition 5] (cf. [SGA4, VIII 9.4.1]). Indeed, $r^{*}$ is exact and conservative, and the Beck-Chevalley condition is verified by smooth base change in case (a) and proper base change in case (b).

A Weil $E_{\lambda}$-sheaf $\mathcal{F}$ on a stack $X$ is called lisse if there exists a smooth presentation $f: Y \rightarrow X$ such that the pullback of $\mathcal{F}$ to $Y \otimes_{\mathbb{F}_{q}} \overline{\mathbb{F}_{q}}$ is isomorphic to $\mathcal{G} \otimes_{\mathcal{O}} E_{\lambda}$ for a lisse $\mathcal{O}$-sheaf $\mathcal{G}$, where $\mathcal{O}$ is the ring of integers of a finite extension of $\mathbb{Q}_{\ell}$ in $E_{\lambda}$. Lemma 1.3 also holds for lisse Weil $E_{\lambda}$-sheaves (and lisse $E_{\lambda}$-sheaves). This follows from the lemma and the following fact.

Lemma 1.4. Let $f: X \rightarrow Y$ be a universally submersive morphism of stacks and let $\mathcal{F}$ be a Weil $E_{\lambda}$-sheaf on $Y$. Then $\mathcal{F}$ is lisse if and only if $f^{*} \mathcal{F}$ is lisse.

Proof. The "only if" part is trivial. To show the "if" part, by taking smooth presentations, we are reduced to the case of schemes (over $\overline{\mathbb{F}_{q}}$ ) and $\mathcal{O}$-sheaves. In this case, the assertion follows from the fact that $f$ is of effective descent for étale morphisms $[\mathbb{R}$, Theorem 5.17].

Remark 1.5. We say that a stack $X$ is geometrically unibranch if for some (or, equivalently, for every) smooth presentation $Y \rightarrow X$, the strict localizations of $Y$ are irreducible. Normal stacks are geometrically unibranch. If $X$ is geometrically unibranch, then every lisse Weil $E_{\lambda}$-sheaf $\mathcal{F}$ satisfies $j_{*} j^{*} \mathcal{F} \simeq \mathcal{F} \otimes j_{*} E_{\lambda} \simeq \mathcal{F}$ for every dominant open immersion $j: U \rightarrow X$. It follows that the pullback of $\mathcal{F}$ to $X \otimes_{\mathbb{F}_{q}} \overline{\mathbb{F}_{q}}$ comes from a lisse $\mathcal{O}$-sheaf.

Let $X$ be a connected stack and let $\bar{x} \rightarrow X$ be a geometric point. The fundamental group $\pi_{1}(X, \bar{x})$ is defined in [N, Section 4], which extends to stacks not necessarily of separated diagonal, as the group of automorphisms of the functor $\mathrm{FEt}(X) \rightarrow$ Fin carrying $Y \rightarrow X$ to (the underlying set of) the geometric fiber $Y \times_{X} \bar{x}$. Here $\operatorname{FEt}(X)$ denotes the category of finit $\AA^{3}$ étale morphisms over $X$, and Fin denotes the category of finite sets. We define the Weil group $W(X, \bar{x})$ to be the inverse image of $W\left(\overline{\mathbb{F}_{q}} / \mathbb{F}_{q}\right)$ under the homomorphism $\pi_{1}(X, \bar{x}) \rightarrow \operatorname{Gal}\left(\overline{\mathbb{F}_{q}} / \mathbb{F}_{q}\right)$. The functor $\operatorname{Shv}_{\text {lisse }}^{W}\left(X, E_{\lambda}\right) \rightarrow \operatorname{Vect}\left(E_{\lambda}\right)$ carrying $\mathcal{F}$ to its stalk $\mathcal{F}_{\bar{x}}$ at $\bar{x}$ is conservative.

If $X$ is connected and geometrically unibranch, then $X$ is irreducible and Weil $E_{\lambda^{-}}$ sheaves (resp. $E_{\lambda}$-sheaves) on $X$ correspond to $E_{\lambda}$-representations of the Weil (resp. fundamental) group of $X$.

Remark 1.6. Let $X$ be a stack. For Weil $E_{\lambda}$-sheaves $\mathcal{F}$ and $\mathcal{G}$ on $X$, we have $\mathcal{F} \simeq \mathcal{G}$ if and only if $\mathcal{F} \otimes_{E_{\lambda}} \overline{\mathbb{Q}_{\ell}} \simeq \mathcal{G} \otimes_{E_{\lambda}} \overline{\mathbb{Q}_{\ell}}$. Similarly, for $A$ and $B$ in the bounded derived category $D^{b}\left(X, E_{\lambda}\right)$ of $E_{\lambda}$-sheaves, $A \simeq B$ if and only if $A \otimes_{E_{\lambda}} \overline{\mathbb{Q}_{\ell}} \simeq B \otimes_{E_{\lambda}} \overline{\mathbb{Q}_{\ell}}$. This follows from Lemma [1.7 below and the fact that rational points form a Zariski dense subset of any affine space over an infinite field (here $E_{\lambda}$ ).

Moreover, a Weil $E_{\lambda}$-sheaf $\mathcal{F}$ on $X$ is an $E_{\lambda}$-sheaf if and only if the Weil $\overline{\mathbb{Q}_{\ell}}$-sheaf $\mathcal{F} \otimes_{E_{\lambda}} \overline{\mathbb{Q}_{\ell}}$ is a $\overline{\mathbb{Q}_{\ell}}$-sheaf. Indeed, we reduce to the case of schemes by Lemma 1.3 and then to lisse sheaves on irreducible geometrically unibranch schemes by Remark 1.2. In this case, the assertion is clear, as the Weil group is dense in the fundamental group.

\footnotetext{
${ }^{3}$ Recall that a morphism of stacks is said to be finite if it is representable by schemes and finite.
} 
For these reasons, we will work mostly with Weil $\overline{\mathbb{Q}_{\ell}}$-sheaves rather than Weil $E_{\lambda^{-}}$ sheaves.

The following is a variant of [SZ, Lemma 2.1.3].

Lemma 1.7. Let $X$ be a stack and let $A$ and $B$ be Weil $E_{\lambda}$-sheaves on $X$ (resp. $A, B \in$ $\left.D^{b}\left(X, E_{\lambda}\right)\right)$. Then there exists a Zariski open subscheme $U=\operatorname{Isom}(A, B)$ of the affine space $\operatorname{Hom}(A, B)$ over $E_{\lambda}$ represented by the $E_{\lambda}$-vector space $\operatorname{Hom}(A, B)$ such that for any algebraic extension $E_{\lambda}^{\prime}$ of $E_{\lambda}$, the set $U\left(E_{\lambda}^{\prime}\right)$ is the set of isomorphisms $A \otimes_{E_{\lambda}} E_{\lambda}^{\prime} \stackrel{\sim}{\rightarrow}$ $B \otimes_{E_{\lambda}} E_{\lambda}^{\prime}$.

Note that $\operatorname{Hom}(A, B)$ is finite-dimensional. Indeed, we have

$$
\operatorname{Hom}(A, B)=\operatorname{Hom}\left(A_{\overline{\mathbb{F}_{q}}}, B_{\overline{\mathbb{F}_{q}}}\right)^{W\left(\overline{\mathbb{F}_{q}} / \mathbb{F}_{q}\right)}
$$

in the case of Weil sheaves, and a short exact sequence

$$
0 \rightarrow \operatorname{Hom}\left(A_{\overline{\mathbb{F}_{q}}}, B_{\overline{\mathbb{F}_{q}}}[-1]\right)_{\operatorname{Gal}\left(\overline{\mathbb{F}_{q}} / \mathbb{F}_{q}\right)} \rightarrow \operatorname{Hom}(A, B) \rightarrow \operatorname{Hom}\left(A_{\overline{\mathbb{F}_{q}}}, B_{\overline{\mathbb{F}_{q}}}\right) \operatorname{Gal}\left(\overline{\mathbb{F}_{q}} / \mathbb{F}_{q}\right) \rightarrow 0
$$

in the case of $D^{b}\left(X, E_{\lambda}\right)$. Here $A_{\overline{\mathbb{F}_{q}}}$ and $B_{\overline{\mathbb{F}_{q}}}$ denote the pullbacks of $A$ and $B$ to $X \otimes_{\mathbb{F}_{q}} \overline{\mathbb{F}_{q}}$. The proof of the lemma is the same as [SZ, Lemma 2.1.3], by taking a finite number of stalk functors.

Following [D2, 1.2.7], for $a \in \overline{\mathbb{Q}}^{\times}$, we let $\overline{\mathbb{Q}}_{\ell}^{(a)}$ denote the Weil $\overline{\mathbb{Q}}_{\ell}$-sheaf on $\operatorname{Spec}\left(\mathbb{F}_{q}\right)$ of rank one such that the geometric Frobenius $\operatorname{Frob}_{q} \in \mathrm{Gal}\left(\overline{\mathbb{F}_{q}} / \mathbb{F}_{q}\right)$ acts by multiplication by $a$. For a stack $X$, we still denote $\pi_{X}^{*} \overline{\mathbb{Q}}^{(a)}$ by $\overline{\mathbb{Q}}_{\ell}^{(a)}$, where $\pi_{X}: X \rightarrow \operatorname{Spec}\left(\mathbb{F}_{q}\right)$ is the projection. We put $\mathcal{F}^{(a)}:=\mathcal{F} \otimes \overline{\mathbb{Q}}^{(a)}$.

The following is an extension to stacks of Deligne's result on determinants [D2, Propositions 1.3.4 (i), 1.3.14] (cf. [D3, 0.4]).

Proposition 1.8. Let $X$ be an irreducible geometrically unibranch stack. Then, for every lisse Weil $\overline{\mathbb{Q}_{\ell}}$-sheaf $\mathcal{F}$ on $X$, there exists a $\in \overline{\mathbb{Q}}_{\ell} \times$ such that $\operatorname{det}\left(\mathcal{F}^{(a)}\right)$ has finite order. Moreover, every simple lisse Weil $\overline{\mathbb{Q}_{\ell}}$-sheaf $\mathcal{F}$ on $X$ such that $\operatorname{det}(\mathcal{F})$ has finite order is $a \overline{\mathbb{Q}_{\ell}}$-sheaf.

Note that $a$ is unique up to multiplication by roots of unity. It follows from the proposition that every simple lisse Weil $\overline{\mathbb{Q}_{\ell}}$-sheaf is the twist of some $\overline{\mathbb{Q}}_{\ell}$-sheaf.

Even if we restrict our attention to $\overline{\mathbb{Q}} \ell$-sheaves, the first part of the proposition is still necessary for the following sections. Our proof of the proposition relies on Lemma 1.11 below, which will be used in later sections as well.

Lemma 1.9. Let $f: X \rightarrow Y$ be a universally submersive morphism of stacks with geometrically connected fibers. Assume $Y$ connected. Then $X$ is connected and the homomorphism $\pi_{1}(X) \rightarrow \pi_{1}(Y)$ induced by $f$ is surjective.

The case of schemes is [SGA1, IX Corollaire 5.6].

Proof. The first assertion follows from the fact that for any quotient map $X \rightarrow Y$ of topological spaces, if $Y$ and the fibers are connected, then $X$ is connected. The second assertion follows from the first one. Indeed, for any connected finite étale cover $Y^{\prime} \rightarrow Y$, $Y^{\prime} \times_{Y} X$ is connected by the first assertion applied to the projection $Y^{\prime} \times_{Y} X \rightarrow Y^{\prime}$.

Lemma 1.10. Any stack $X$ admits a dense open substack with separated diagonal. 
Proof. There exists a dense open substack $V$ of $X$ with flat inertia. Then $V$ is a gerbe over an algebraic space [SP, Tag 06QJ]. Since any group algebraic space of finite presentation over a field is separated (and in fact a group scheme, see [B, Proposition 5.1.1] or [SP, Tags 08BH, 0B8G]), there exists a dense open substack $U$ of $V$ with separated inertia. Then $U$ has separated diagonal by fppf descent [SP, Tags 0CPS, 0DN6].

Lemma 1.11. Let $\mathcal{F}$ be a lisse Weil $\overline{\mathbb{Q}_{\ell}}$-sheaf on a stack $X$. Then there exists a dominant open immersion $j: U \rightarrow X$ and a gerbe-like morphism $f: U \rightarrow Y$, where $Y$ is a DeligneMumford stack, such that the adjunction map $f^{*} \mathcal{G} \rightarrow j^{*} \mathcal{F}$, where $\mathcal{G}:=f_{*} j^{*} \mathcal{F}$, is an isomorphism.

Recall that any gerbe-like morphism $f: U \rightarrow Y$ of stacks is a smooth universal homeomorphism [SP, Tags 06R9, 0DN8]. Note that $f^{*} \mathcal{G} \simeq j^{*} \mathcal{F}$ implies that $\mathcal{G}$ is a lisse Weil $\overline{\mathbb{Q}_{\ell}}$-sheaf, which is simple if $j^{*} \mathcal{F}$ is simple. Moreover, if $X$ is geometrically unibranch, then $Y$ is geometrically unibranch, and $\operatorname{det}(\mathcal{F})$ and $\operatorname{det}(\mathcal{G})$ have the same (possibly infinite) order by Lemma 1.9.

Proof. By a dévissage result of Behrend [B, Propositions 5.1.11, 5.1.14], there exists a dominant open immersion $j: U \rightarrow X$ and a gerbe-like morphism $f: U \rightarrow Y$, where $Y$ is a Deligne-Mumford stack, such that the diagonal of $f$ has connected geometric fibers. By generic base change [IZ, Proposition 2.11], up to shrinking $Y$ (and $U$ ), we may assume that $f_{*} \mathcal{F}$ commutes with base change. It then suffices to check that the adjunction $f_{y}^{*} f_{y *}\left(\mathcal{F} \mid U_{y}\right) \rightarrow \mathcal{F} \mid U_{y}$ is an isomorphism for every geometric fiber $f_{y}: U_{y} \rightarrow y$ of $f$. Since $U_{y}$ is the classifying stack of a connected group scheme over $y$, any sheaf on $U_{y}$ is constant and the assertion is trivial.

Remark 1.12. Since any gerbe over a finite field is neutral [B, Corollary 6.4.2], any point $y \in Y\left(\mathbb{F}_{q^{n}}\right)$ lifts to a point of $U\left(\mathbb{F}_{q^{n}}\right)$. In particular, $E(\mathcal{G})=E\left(j^{*} \mathcal{F}\right)$.

Proof of Proposition 1.8. Applying Lemma 1.11, we are reduced to the case where $X$ is a Deligne-Mumford stack. Here for the second assertion of the proposition, we have used the fact that $\mathcal{F} \simeq j_{*} j^{*} \mathcal{F}$. By Lemma 1.10] and [LMB, Corollaire 6.1.1], up to shrinking $X$, we may assume $X=[Y / G]$, where $Y$ is a separated scheme and $G$ is a finite group acting on $Y$. Up to replacing $Y$ by a connected component and $G$ by the decomposition group, we may assume that $Y$ is irreducible. Let $g: Y \rightarrow X$. Then $g^{*} \mathcal{F}$ corresponds to the restriction to the open normal subgroup $\pi_{1}(Y) \triangleleft \pi_{1}(X)$ of quotient $G$. By the case of schemes of the first assertion [D2, Proposition 1.3.4 (i)], there exists $a \in \overline{\mathbb{Q}}_{\ell} \times$ such that $\operatorname{det}\left(g^{*} \mathcal{F}^{(a)}\right)^{\otimes n} \simeq \overline{\mathbb{Q}_{\ell}}$ for some $n \geq 1$. Then $\operatorname{det}\left(\mathcal{F}^{(a)}\right)^{\otimes(n \cdot \# G)} \simeq \overline{\mathbb{Q}_{\ell}}$. This finishes the proof of the first assertion of Proposition [1.8. Now assume that $\mathcal{F}$ is simple and $\operatorname{det}(\mathcal{F})$ has finite order. By Lemma 1.13 below and the case of schemes of the second assertion of Proposition [1.8 [D2, Proposition 1.3.14], $g^{*} \mathcal{F}$ is a $\overline{\mathbb{Q}_{\ell}}$-sheaf, so that the same holds for $\mathcal{F}$ by Lemma 1.3 .

Lemma 1.13. Let $f: X \rightarrow Y$ be a finite étale morphism of geometrically unibranch stacks and let $\mathcal{F}$ be a simple lisse Weil $\overline{\mathbb{Q}_{\ell}}$-sheaf on $Y$ such that $\operatorname{det}(\mathcal{F})$ has finite order. Then $f^{*} \mathcal{F} \simeq \bigoplus_{i} \mathcal{F}_{i}$ is semisimple with simple factors $\mathcal{F}_{i}$ such that each $\operatorname{det}\left(\mathcal{F}_{i}\right)$ has finite order.

Proof. We may assume that $X$ and $Y$ are irreducible. Since $\pi_{1}(X) \subseteq \pi_{1}(Y)$ is an open subgroup of finite index, $f^{*} \mathcal{F} \simeq \bigoplus_{i} \mathcal{F}_{i}$ is semisimple. For the assertion on simple factors, we may assume that $f$ is a Galois cover of group $G$. Fix an $i_{0}$. By the first assertion of Proposition [1.8, there exists $a \in \overline{\mathbb{Q}}_{\ell} \times$ such that $\operatorname{det}\left(\mathcal{F}_{i_{0}}^{(a)}\right)$ has finite order. Since the 
simple factors are permuted transitively by $G$, $\operatorname{det}\left(\mathcal{F}_{i}^{(a)}\right)$ has finite order for each $i$. It follows that $\operatorname{det}\left(\mathcal{F}^{(a)}\right) \simeq \bigotimes_{i} \operatorname{det}\left(\mathcal{F}_{i}^{(a)}\right)$ has finite order. This implies that $a$ is a root of unity. Therefore, each $\operatorname{det}\left(\mathcal{F}_{i}\right)$ has finite order.

Remark 1.14. (1) In the above proof of Proposition 1.8, we first reduce to the case of quotient stacks $[Y / G]$ by finite groups $G$ by Lemma 1.11, and then reduce to the case of schemes. The same strategy will be used for Theorems 0.1 , 0.2, and 2.6. Another approach is to use the generic existence of a smooth presentation with geometrically connected fibers ([LMB, Théorème 6.5] and Lemma [1.10) to reduce directly to the case of schemes, which works for Proposition 1.8 and Theorems 0.1 (1) and 2.6, but fails for Theorems 0.1 (2) and 0.2.

(2) The reduction from quotient stacks $[Y / G]$ to schemes here and in Theorem 0.1 (1) uses Lemma 1.13 applied to the finite étale cover $Y \rightarrow[Y / G]$. We may replace this by Lemma 1.9 applied to the $\mathrm{GL}_{m}$-torsor $Y \wedge^{G} \mathrm{GL}_{m} \rightarrow[Y / G]$, where $G \rightarrow \mathrm{GL}_{m}$ is a chosen embedding, making the proofs closer to those of Theorems 0.1 (2), 0.2, and 2.6.

(3) We can also prove Proposition 1.8 directly by imitating the proof of the case of schemes. Indeed, as in [D2, Proposition 1.3.4, Variante], the first assertion follows from the case of curves by joining by curves (see the proof of Proposition 2.2) and Chebotarev's density theorem (Proposition 4.6). As in [D2, Theorem 1.3.8], this implies that the radical of $G^{00}$ is unipotent, where $G^{00}$ is the identity component of the geometric monodromy group, which is a theorem of Grothendieck in the case of schemes. Finally, as in [D2, Proposition 1.3.14], the second assertion follows from this and the first assertion.

Proposition 1.8 has the following consequence on the structure of Weil $\overline{\mathbb{Q}} \ell^{-}$-sheaves. For a stack $X$, we let $\operatorname{Shv}\left(X, \overline{\mathbb{Q}_{\ell}}\right)^{(a)} \subseteq \operatorname{Shv}^{W}\left(X, \overline{\mathbb{Q}_{\ell}}\right)$ denote the full subcategory spanned by Weil $\overline{\mathbb{Q}_{\ell}}$-sheaves of the form $\mathcal{F}^{(a)}$ with $\mathcal{F} \in \operatorname{Shv}\left(X, \overline{\mathbb{Q}_{\ell}}\right)$. The subcategory only depends on the class of $a$ in $\overline{\mathbb{Q}}_{\ell} \times / \overline{\mathbb{Z}}_{\ell} \times$, where $\overline{\mathbb{Z}_{\ell}}$ denotes the ring of integers of $\overline{\mathbb{Q}_{\ell}}$.

Proposition 1.15. Let $X$ be a stack. We have a canonical decomposition:

$$
\operatorname{Shv}^{W}\left(X, \overline{\mathbb{Q}_{\ell}}\right) \simeq \underset{a \in \overline{\mathbb{Q}}_{\ell}^{\times} / \overline{\mathbb{Z}}_{\ell} \times}{ } \operatorname{Shv}\left(X, \overline{\mathbb{Q}_{\ell}}\right)^{(a)}
$$

Proof. It suffices to show the following:

- (generation) Every object of $\operatorname{Shv}^{W}\left(X, \overline{\mathbb{Q}_{\ell}}\right)$ is a successive extension of objects of $\operatorname{Shv}\left(X, \overline{\mathbb{Q}_{\ell}}\right)^{(a)}$;

- (orthogonality) $\operatorname{Ext}^{i}\left(A^{(a)}, B^{(b)}\right)=0$ for $A, B \in \operatorname{Shv}\left(X, \overline{\mathbb{Q}_{\ell}}\right), a / b \notin \overline{\mathbb{Z}}_{\ell} \times$ and $i=0,1$.

The first point follows from Proposition 1.8. Let us show the orthogonality. We have $\operatorname{Hom}\left(A^{(a)}, B^{(b)}\right)=\operatorname{Hom}\left(A_{\mathbb{F}_{q}}^{(a)}, B_{\mathbb{F}_{q}}^{(b)}\right)^{W\left(\overline{\mathbb{F}_{q}} / \mathbb{F}_{q}\right)}$ and a short exact sequence (Remark $1.2(1)$,

$$
0 \rightarrow \operatorname{Hom}\left(A_{\overline{\mathbb{F}_{q}}}^{(a)}, B_{\overline{\mathbb{F}_{q}}}^{(b)}\right)_{W\left(\overline{\mathbb{F}_{q}} / \mathbb{F}_{q}\right)} \rightarrow \operatorname{Ext}^{1}\left(A^{(a)}, B^{(b)}\right) \rightarrow \operatorname{Hom}\left(A_{\overline{\mathbb{F}_{q}}}^{(a)}, B_{\overline{\mathbb{F}_{q}}}^{(b)}[1]\right)^{W\left(\overline{\mathbb{F}_{q}} / \mathbb{F}_{q}\right)} \rightarrow 0 .
$$

The $\overline{\mathbb{Q}_{\ell}}$-vector space $\operatorname{Hom}\left(A_{\mathbb{F}_{q}}^{(a)}, B_{\overline{\mathbb{F}_{q}}}^{(b)}[i]\right)$ with $W\left(\overline{\mathbb{F}_{q}} / \mathbb{F}_{q}\right)$-action can be identified with the

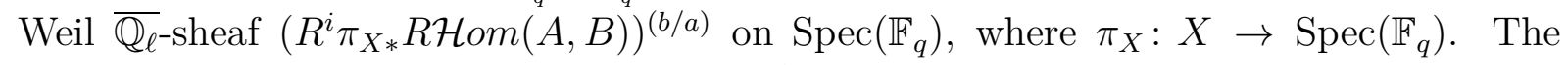
eigenvalues of Frob $_{q}$ are all in the class of $b / a$, so the action has no nonzero invariants or coinvariants. Therefore, $\operatorname{Ext}^{i}\left(A^{(a)}, B^{(b)}\right)=0$ for $i=0,1$. 
Corollary 1.16. Let $X$ be a stack. A Weil $\overline{\mathbb{Q}_{\ell}}$-sheaf $\mathcal{F}$ is a $\overline{\mathbb{Q}_{\ell}}$-sheaf if and only if for every $x \in X\left(\mathbb{F}_{q^{n}}\right), n \geq 1$, the eigenvalues of Frob $_{x}$ acting on $\mathcal{F}_{\bar{x}}$ are $\ell$-adic units.

By reducing to curves (see Proposition 2.2 below), we see that for lisse Weil $\overline{\mathbb{Q}_{\ell^{-}}}$ sheaves, it suffices to check the condition in the corollary for one given $x$ in each connected component of $X$.

\section{Frobenius eigenvalues}

In this section, we prove Theorem 0.1(1) on Frobenius eigenvalues. We then extend the theorem of Drinfeld and Kedlaya on Newton polygons from smooth schemes to normal stacks (Theorem [2.6). Finally, following Drinfeld [D4, Appendix B], we study the category of weakly motivic complexes, whose cohomology sheaves have "motivic" Frobenius eigenvalues (Theorems 2.13 and 2.15).

Notation 2.1. We let $M_{0}$ denote the group of algebraic numbers $\alpha \in \overline{\mathbb{Q}}^{\times}$of weight 0 relative to $q$, namely, such that for every Archimedean place $\lambda$ of $\mathbb{Q}(\alpha)$, we have $|\alpha|_{\lambda}=1$. We let $W_{0}(q) \subseteq M_{0}$ denote the subgroup of $q$-Weil numbers of weight 0 . For a subset $S \subseteq \mathbb{Q}$ of slopes, we let $W_{0}^{S}(q) \subseteq W_{0}(q)$ denote the subgroup of $\alpha \in W_{0}(q)$ of slopes in $S$, namely such that for every valuation $v$ on $\mathbb{Q}(\alpha)$ satisfying $v(q)=1$, we have $v(\alpha) \in S$.

Note that $W_{0}(q)$ only depends on the characteristic $p$ of $\mathbb{F}_{q}$ and that $W_{0}^{\{0\}}(q)$ is simply the set of roots of unity in $\overline{\mathbb{Q}}$.

In the notation above, Theorem 0.1 (1) says that the eigenvalues of Frob ${ }_{x}$ acting on $\mathcal{F}_{\bar{x}}$ belong to $W_{0}^{\left[-\frac{r-1}{2}, \frac{r-1}{2}\right] \cap \mathbb{Q}}\left(q^{n}\right)$ for all $x \in X\left(\mathbb{F}_{q^{n}}\right)$ and all $n \geq 1$. As mentioned earlier, we prove this by reducing to the case of schemes. For the reduction to work, we need to show that the statement can be checked on any dense open substack. We start by reviewing Deligne's argument of joining by curves [D3, Proposition 1.9] and extending it to stacks.

Proposition 2.2. Let $X$ be a connected stack. Then there exists an integer $M \geq 1$ such that, for every lisse Weil $\overline{\mathbb{Q}_{\ell}}$-sheaf $\mathcal{F}$ on $X$ of rank $r \geq 1$, and for all $m, n \geq 1$, $x \in X\left(\mathbb{F}_{q^{m}}\right), y \in X\left(\mathbb{F}_{q^{n}}\right)$, if we let $\alpha_{1}, \ldots, \alpha_{r}$ (resp. $\beta_{1}, \ldots, \beta_{r}$ ) denote the eigenvalues of Frob $_{x}(\text { resp. Frob })_{y}$ ) acting on $\mathcal{F}_{\bar{x}}\left(\right.$ resp. $\left.\mathcal{F}_{\bar{y}}\right)$, then, up to reordering, we have $\beta_{i}^{1 / n} / \alpha_{i}^{1 / m} \in$ $W_{0}^{[-M(r-1), M(r-1)] \cap \mathbb{Q}}(q)$ for $1 \leq i \leq r$.

In the situation of the proposition, if $\alpha_{1}, \ldots, \alpha_{r} \in W_{0}(q)$, then $\beta_{1}, \ldots, \beta_{r} \in W_{0}(q)$.

Proof. Let $f: Y \rightarrow X$ be a submersive morphism (for example, a flat presentation) with $Y$ a separated algebraic space. Consider the intersection graph $\Gamma$ of the irreducible components of $Y$ over $X$. The vertices are the irreducible components of $Y$. There is an edge between two vertices $v$ and $w$ if and only if the corresponding components $Y_{v}$ and $Y_{w}$ are such that $Y_{v} \times_{X} Y_{w}$ is nonempty. If $Y_{v}$ and $Y_{w}$ are on the same connected component of $Y$, then $v$ and $w$ belong to the same connected component of $\Gamma$. Thus for each component $V$ of $\Gamma, Y_{V}:=\bigcup_{v \in V} Y_{v}$ is a union of connected components of $Y$. It follows that $f\left(Y_{V}\right)$ is open and closed, because $f^{-1}\left(f\left(Y_{V}\right)\right)=Y_{V}$. Since $X$ is connected, so is $\Gamma$.

We take $M-1$ to be the diameter of the graph $\Gamma$. For each edge $e=(v, w)$ of the graph, choose a closed point $x_{e}$ of $Y_{v} \times_{X} Y_{w}$. For $x$ and $y$ as in the statement of the proposition, let $v$ and $w$ be vertices such that $\bar{x}$ lifts to $Y_{v}$ and $\bar{y}$ lifts to $Y_{w}$. Let 
$v=v_{1} \frac{e_{1}}{1} v_{2} \cdots v_{N-1} \frac{e_{N-1}}{\frac{1}{2}} v_{N}=w$ be a path of length $N-1 \leq M-1$. By Lemma 2.3 below, there exists a diagram above $X$

$$
x_{0} \rightarrow C_{1} \leftarrow x_{1} \rightarrow \cdots \leftarrow x_{N-1} \rightarrow C_{N} \leftarrow x_{N},
$$

where $C_{j}, 1 \leq j \leq N$ are irreducible smooth curves over $\mathbb{F}_{q}$ above $Y_{v_{j}}$, and $x_{j}=$ $\operatorname{Spec}\left(\mathbb{F}_{q^{n_{j}}}\right), 0 \leq j \leq N$ such that $x_{0}$ is above $x, x_{j}$ is above $x_{e_{j}}$ for $1 \leq j \leq N-1$, and $x_{N}$ is above $y$. We apply the proof of L. Lafforgue's theorem [L1, Théorème VII.6] (or V. Lafforgue's improvement of the bound [L2, Corollaire 2.2]) to $C_{j}, 1 \leq j \leq N$, and to the simple factors of the pullback of $\mathcal{F}$ to $C_{j}$. If $\alpha_{1}^{(j)}, \ldots, \alpha_{r}^{(j)}$ denote the eigenvalues of Frob $_{x_{j}}$, then up to reordering the $r$ values, we have $\left(\alpha_{i}^{(j)}\right)^{1 / n_{j}} /\left(\alpha_{i}^{(j-1)}\right)^{1 / n_{j-1}} \in W_{0}^{[-(r-1),(r-1)]}(q)$.

Lemma 2.3. Let $Y$ be an irreducible algebraic space separated of finite type over a field $k$ and let $x$ and $y$ be closed points of $Y$. There exists an irreducible regular curve $C$ and $a$ morphism $C \rightarrow Y$ of image containing $x$ and $y$.

Proof. By Chow's lemma [K, IV Theorem 3.1], we may assume that $Y$ is a scheme. Replacing $Y$ by an irreducible component of $Y \otimes_{k} \bar{k}$, we may assume that $k$ is algebraically closed. For this case, see [M, Section 6].

Proposition 2.4. Let $R$ be an integrally closed subring of $\overline{\mathbb{Q}_{\ell}}$. Let $j: U \rightarrow X$ be a dominant open immersion of stacks and let $\mathcal{F}$ be a lisse Weil $\overline{\mathbb{Q}_{\ell}}$-sheaf on $X$ such that $j^{*} \mathcal{F}$ is $R$-integral (resp. inverse $R$-integral). Then $\mathcal{F}$ is $R$-integral (resp. inverse $R$-integral).

Recall that, following [Z2, Variante 5.13, Définition 6.1], a Weil $\overline{\mathbb{Q}_{\ell}}$-sheaf $\mathcal{F}$ on $X$ is said to be $R$-integral (resp. inverse $R$-integral) if for all $n \geq 1$ and all $x \in X\left(\mathbb{F}_{q^{n}}\right)$, the eigenvalues (resp. inverse eigenvalues) of Frob $x$ acting on $\mathcal{F}_{\bar{x}}$ are in $R$.

Proof. Up to replacing $X$ by a smooth presentation, we may assume that $X$ is a scheme. Up to replacing $X$ by its normalization, we may further assume that $X$ is normal. In this case, $\mathcal{F} \simeq j_{*} j^{*} \mathcal{F}$ is $R$-integral (resp. inverse $R$-integral) by [Z2, Théorème 2.2, Variantes 5.1, 5.13]. (The integral case is a theorem of Deligne [SGA7II, XXI Théorème 5.6] assuming resolution of singularities.)

Remark 2.5. Let $I=[a, b] \cap \mathbb{Q}$ be an interval with $a, b \in \mathbb{Q}$. It follows from the propositions that if $\mathcal{F}$ is a lisse $\overline{\mathbb{Q}_{\ell}}$-sheaf on a stack $X$ such that the eigenvalues of Frob $x$ acting on $\mathcal{F}_{\bar{x}}$ belong to $W_{0}^{I}\left(q^{n}\right)$ for all $n \geq 1$ and all $x \in U\left(\mathbb{F}_{q^{n}}\right)$, where $U$ is some dense open substack of $X$, then the same holds for all $x \in X\left(\mathbb{F}_{q^{n}}\right)$. Indeed, the eigenvalues belong to $W_{0}(q)$ by Proposition 2.2 , and $\mathcal{F}^{\left(q^{-a}\right)}$ is $\overline{\mathbb{Z}}$-integral and $\mathcal{F}^{\left(q^{-b}\right)}$ is inverse $\overline{\mathbb{Z}}$ integral (for all representatives of $q^{-a}$ and $q^{-b}$ ) by Proposition 2.4. Here $\overline{\mathbb{Z}}$ denotes the ring of algebraic integers.

Proof of Theorem 0.1 (1). By Remark 2.5, we may shrink $X$. Thus, by Lemma 1.11, we are reduced to the case of Deligne-Mumford stacks. Up to shrinking $X$, we may assume that there exists a finite étale cover $f: Y \rightarrow X$, where $Y$ is a scheme. By Lemma 1.13, $f^{*} \mathcal{F} \simeq \bigoplus_{i} \mathcal{F}_{i}$, with $\mathcal{F}_{i}$ simple and $\operatorname{det}\left(\mathcal{F}_{i}\right)$ of finite order. We are thus reduced to the case where $X$ is a scheme. This case was stated in [L1, Proposition VII.7], and the gap in the proof has been fixed by Deligne [D3, Théorème 1.6] and others. Indeed, by Remark 2.5] again, we may assume that $X$ is a smooth separated scheme. By a consequence of Hilbert irreducibility ([D4, Proposition 2.17] or [EK, Proposition B.1]), for any closed point $x$ of $X$, there exists a smooth curve $C$ over $\mathbb{F}_{q}$ and a morphism $g: C \rightarrow X$ such that $x$ is in 
the image of $g$ and $g^{*} \mathcal{F}$ is simple. It then suffices to apply L. Lafforgue's theorem for curves [L1, Théorème VII.6] and V. Lafforgue's improvement of the bound [L2, Corollaire $2.2]$.

More generally V. Lafforgue proved an inequality for the Newton polygon in the case of curves. Recently Drinfeld and Kedlaya [DK, Theorem 1.3.3] gave a refinement for the lowest Newton polygon in the case of smooth schemes. These results extend to normal stacks as follows.

For a stack $X$, we let $\left|X\left(\overline{\mathbb{F}_{q}}\right)\right|$ denote the set of isomorphism classes of the groupoid $X\left(\overline{\mathbb{F}_{q}}\right)$. We let $|X|$ denote the set of orbits of $\operatorname{Gal}\left(\overline{\mathbb{F}_{q}} / \mathbb{F}_{q}\right)$ acting on $\left|X\left(\overline{\mathbb{F}_{q}}\right)\right|$. If $X$ is a Deligne-Mumford stack, then $|X|$ can be identified with the set of closed points of $X$. In general, following [DK, Lemma 5.3.4], we equip $|X|$ with the following topology $T$ : a subset $U \subseteq|X|$ is $T$-open if and only if for every morphism $C \rightarrow X$ from a smooth curve $C$ to $X$, the inverse image of $U$ under the map $|C| \rightarrow|X|$ is open for the Zariski topology on $|C|$.

We say that a Weil $\overline{\mathbb{Q}}$-sheaf $\mathcal{F}$ on $X$ is algebraic if it is $\overline{\mathbb{Q}}$-integral. We fix a valuation $v$ on $\overline{\mathbb{Q}}$ such that $v(q)=1$. For an algebraic Weil $\overline{\mathbb{Q}} \ell^{-}$sheaf $\mathcal{F}$ on $X$ and $x \in X\left(\mathbb{F}_{q^{n}}\right)$, we let $s_{1}^{x}(\mathcal{F}) \leq \cdots \leq s_{r}^{x}(\mathcal{F})$ denote the images under $v / n$ of the eigenvalues of Frob $_{x}$ acting on $\mathcal{F}_{\bar{x}}$. These rational numbers are called the slopes of $\mathcal{F}$ at $x$ and depend on $x$ only through the image of $x$ in $|X|$.

Theorem 2.6. Let $X$ be an irreducible stack. Let $\mathcal{F}$ be an algebraic lisse Weil $\overline{\mathbb{Q}_{\ell}}$-sheaf of rank $r$ on $X$. Then

(1) There exist rational numbers $s_{1}(\mathcal{F}) \leq \cdots \leq s_{r}(\mathcal{F})$ such that $\sum_{j=1}^{i} s_{j}(\mathcal{F}) \leq \sum_{j=1}^{i} s_{j}^{x}(\mathcal{F})$ for all $x$ and all $i$ and the set $Y \subseteq|X|$ of $y$ satisfying $s_{i}^{y}(\mathcal{F})=s_{i}(\mathcal{F})$ for all $i$ is nonempty and T-open.

(2) If $X$ is geometrically unibranch and $\mathcal{F}$ is indecomposable, then $s_{i+1}(\mathcal{F}) \leq s_{i}(\mathcal{F})+1$ for all $1 \leq i \leq r-1$.

The numbers $s_{i}(\mathcal{F})$ are the slopes of the lowest Newton polygon.

Remark 2.7. In (2), if moreover $\operatorname{det}(\mathcal{F})$ has finite order, so that $\sum_{j=1}^{r} s_{j}^{x}(\mathcal{F})=0$, then, as in [DK, Proof of Corollary 1.1.7], the theorem implies

$$
\sum_{j=1}^{i} s_{j}^{x}(\mathcal{F}) \geq \sum_{j=1}^{i} s_{j}(\mathcal{F}) \geq-i(r-i) / 2
$$

for all $x$ and all $i$. Taking $i=1$ and $i=r-1$, we recover the bounds $s_{1}^{x}(\mathcal{F}) \geq-(r-1) / 2$ and $s_{r}^{x}(\mathcal{F}) \leq(r-1) / 2$ in Theorem $0.1(1)$.

To prove the theorem, we need a couple of lemmas, extending [DK, Lemmas 5.3.1, 5.3.3, 5.3.4].

Lemma 2.8. Let $X$ be a stack and let $\mathcal{F}$ be an algebraic lisse Weil $\overline{\mathbb{Q}_{\ell}}$-sheaf on $X$. For all $i$, the function $x \mapsto \sum_{j=1}^{i} s_{j}^{x}(\mathcal{F})$ on $|X|$ is upper semi-continuous for the topology $T$, bounded, and takes values in $N^{-1} \mathbb{Z}$ for some $N$.

Proof. By the definition of the topology $T$, for the semi-continuity we may assume that $X$ is a smooth curve. We reduce then to the case $\mathcal{F}$ simple, and then to the case where $\operatorname{det}(\mathcal{F})$ has finite order. In this case, the semi-continuity follows from Abe's theorem on crystalline 
companions [A, Theorem 4.4.1] and the corresponding statement for overconvergent $F$ isocrystals. The boundedness follows from Proposition 2.2. The last assertion follows from the fact that $E(\mathcal{F})$ is a number field (Theorem 3.1) by the proof of [DK, Lemma 5.3.1].

Lemma 2.9. Let $X$ be an irreducible stack. Then $|X|$ is irreducible for the topology $T$.

Proof. If $f: Y \rightarrow X$ is a surjective morphism of stacks with $Y$ irreducible, then $|f|$ is a surjection and we may replace $X$ by $Y$. Thus, replacing $X$ by its normalization, we may assume $X$ is normal. Next note that if $X$ admits a Zariski open cover $\left(X_{i}\right)$ such that $\left|X_{i}\right|$ is $T$-irreducible for all $i$, then $X$ is $T$-irreducible. Indeed, $\left|X_{i}\right| \cap\left|X_{j}\right| \neq \emptyset$ and [EGAI, 0 2.1.4] applies. Let $f: Y \rightarrow X$ be a flat presentation with $Y$ a separated scheme. Each connected component $Y_{i}$ of $Y$ is irreducible, and $\left(f\left(Y_{i}\right)\right)$ is a Zariski open cover of $X$. We may thus replace $X$ by $Y_{i}$, and assume that $X$ is a separated scheme. Let $U_{1}, U_{2} \subseteq|X|$ be nonempty $T$-open subsets. Let $x_{i} \in U_{i}$. By Lemma 2.3, there exists a morphism $g: C \rightarrow X$, where $C$ is an irreducible smooth curve, such that $x_{1}$ and $x_{2}$ are in the image of $|g|$. Then $|g|^{-1}\left(U_{i}\right)$ is nonempty for $i=1,2$. It follows that $|g|^{-1}\left(U_{1} \cap U_{2}\right)$ and hence $U_{1} \cap U_{2}$ are nonempty.

Proof of Theorem [2.6. (1) By Lemma 2.8, the function $a_{i}: x \mapsto \sum_{j=1}^{i} s_{j}^{x}(\mathcal{F})$ on $|X|$ attains a minimum. We define $s_{1} \leq \cdots \leq s_{r}$ so that the minimum of $a_{i}$ is $\sum_{j=1}^{i} s_{j}$. Moreover, the locus $Y_{i} \subseteq|X|$ on which $a_{i}$ attains the minimum is $T$-open. Therefore, $Y=\bigcap_{i=1}^{r-1} Y_{i}$ is nonempty and $T$-open by Lemma 2.9 ,

(2) Since $|X|$ is irreducible, we may shrink $X$. Thus, by Lemma 1.11, we may assume that $X$ is a Deligne-Mumford stack. Further shrinking $X$, we may assume that $X=$ $[Y / G]$ is the quotient stack of a smooth affine scheme $Y$ by a finite group $G$. Choose an embedding $G \rightarrow \mathrm{GL}_{m}$. Then $Y \wedge^{G} \mathrm{GL}_{m}=\left(Y \times \mathrm{GL}_{m}\right) / G$ is a $\mathrm{GL}_{m}$-torsor over $[Y / G]$. By Lemma 1.9, we may replace $X$ by the smooth affine scheme $Y \wedge^{G} \mathrm{GL}_{m}$. This case is DK, Theorem 1.3.3].

Let $\iota: \overline{\mathbb{Q}_{\ell}} \rightarrow \mathbb{C}$ be an embedding. Following [S1, 2.4.3], we say that a Weil $\overline{\mathbb{Q}_{\ell}}$-sheaf $\mathcal{F}$ on a stack $X$ is punctually $\iota$-pure of weight $w \in \mathbb{R}$ if for every $x \in X\left(\mathbb{F}_{q^{n}}\right), n \geq 1$ and every eigenvalue $\alpha$ of $\operatorname{Frob}_{x}$ acting on $\mathcal{F}_{\bar{x}}$, we have $|\iota \alpha|=q^{w n / 2}$. The results of Sun in [S1] and [S2] extend to stacks not necessarily of separated diagonal by Lemma 1.10 and to Weil $\overline{\mathbb{Q}_{\ell}}$-sheaves by Proposition 1.15 , For $w \in \mathbb{Z}$, we say that $\mathcal{F}$ is punctually pure of weight $w$ if it is punctually $\iota$-pure of weight $w$ for all $\iota$.

Remark 2.10. By Theorem 0.1 (1) and Proposition 1.8, every simple lisse Weil $\overline{\mathbb{Q}_{\ell}}$-sheaf on a geometrically unibranch stack is punctually $\iota$-pure. It follows that every Weil $\overline{\mathbb{Q}_{\ell^{-}}}$ sheaf on a stack is $\iota$-mixed, namely, a successive extension of punctually $\iota$-pure sheaves (cf. [S1, Remark 2.8.1]). Similarly, a Weil $\overline{\mathbb{Q}_{\ell}}$-sheaf $\mathcal{F}$ on a stack is mixed, namely, a successive extension of punctually pure sheaves (of integral weights), if and only if for every $x \in X\left(\mathbb{F}_{q^{n}}\right), n \geq 1$, the eigenvalues of Frob $x$ acting on $\mathcal{F}_{\bar{x}}$ belong to $M\left(q^{n}\right)$. Here $M(q):=\bigcup_{w \in \mathbb{Z}} q^{w / 2} M_{0}$. (Recall that $M_{0}$ is the group of algebraic numbers of weight 0 .)

The structure of punctually $\iota$-pure Weil $\overline{\mathbb{Q}_{\ell}}$-sheaves can be described as follows. We let $\mathcal{E}_{n}$ denote the $\overline{\mathbb{Q}}_{\ell}$-sheaf on $\operatorname{Spec}\left(\mathbb{F}_{q}\right)$ of stalk $\overline{\mathbb{Q}}_{\ell}^{n}$ on which Frob $q$ acts unipotently with one Jordan block.

Proposition 2.11. Let $X$ be a geometrically unibranch stack. Then indecomposable punctually ı-pure lisse Weil $\overline{\mathbb{Q}_{\ell}}$-sheaves are of the form $\mathcal{F} \otimes \pi_{X}^{*} \mathcal{E}_{n}$ with $\mathcal{F}$ simple, where $\pi_{X}: X \rightarrow \operatorname{Spec}\left(\mathbb{F}_{q}\right)$. 
In the appendix we will prove an analogue for pure perverse sheaves. The proposition still holds with $\overline{\mathbb{Q}_{\ell}}$ replaced by a finite (or algebraic) extension of $\mathbb{Q}_{\ell}$.

Proof. As in the case of perverse sheaves on schemes [BBD, Proposition 5.3.9 (i)], this follows from the geometric semisimplicity of punctually $\iota$-pure lisse Weil $\overline{\mathbb{Q}} \ell^{-}$-sheaves $[\mathrm{S} 2$, Theorem 2.1 (iii)] (generalizing [D2, Théorème 3.4.1 (iii)]).

Let $W(q)=\bigcup_{w \in \mathbb{Z}} q^{w / 2} W_{0}(q)$ be the group of $q$-Weil numbers (of integral weights). We say that $K \in D\left(X, \overline{\mathbb{Q}_{\ell}}\right)$ is weakly motivic if for all $n \geq 1, x \in X\left(\mathbb{F}_{q^{n}}\right)$, and $i \in$ $\mathbb{Z}$, the eigenvalues of Frob ${ }_{x}$ acting on $\mathcal{H}^{i} K_{\bar{x}}$ belong to $W\left(q^{n}\right)$. We let $D_{\text {mot }}\left(X, \overline{\mathbb{Q}_{\ell}}\right) \subseteq$ $D\left(X, \overline{\mathbb{Q}_{\ell}}\right)$ denote the full subcategory spanned by weakly motivic complexes, which is a thick subcategory. For $* \in\{+,-, b\}$, we put $D_{\text {mot }}^{*}=D^{*} \cap D_{\text {mot }}$.

Remark 2.12. By Proposition 2.2 , for a lisse $\overline{\mathbb{Q}_{\ell}}$-sheaf $\mathcal{F}$ on a connected stack $X$ and a fixed $x \in X\left(\mathbb{F}_{q^{n}}\right), \mathcal{F}$ is weakly motivic if and only if the eigenvalues of Frob $x$ acting on $\mathcal{F}_{\bar{x}}$ are in $W\left(q^{n}\right)$.

The following result generalizes [D4, Theorems B.3, B.4].

Theorem 2.13. Let $f$ be a morphism of stacks. The six operations and GrothendieckVerdier duality induce

$$
\begin{gathered}
\otimes: D_{\mathrm{mot}}^{-}\left(X, \overline{\mathbb{Q}_{\ell}}\right) \times D_{\mathrm{mot}}^{-}\left(X, \overline{\mathbb{Q}_{\ell}}\right) \rightarrow D_{\mathrm{mot}}^{-}\left(X, \overline{\mathbb{Q}_{\ell}}\right), \\
R \mathcal{H o m}: D_{\mathrm{mot}}^{-}\left(X, \overline{\mathbb{Q}_{\ell}}\right)^{\mathrm{op}} \times D_{\mathrm{mot}}^{+}\left(X, \overline{\mathbb{Q}_{\ell}}\right) \rightarrow D_{\mathrm{mot}}^{+}\left(X, \overline{\mathbb{Q}_{\ell}}\right), \\
D: D_{\mathrm{mot}}\left(X, \overline{\mathbb{Q}_{\ell}}\right)^{\mathrm{op}} \rightarrow D_{\mathrm{mot}}\left(X, \overline{\mathbb{Q}_{\ell}}\right), \quad f^{*}, f^{!}: D_{\mathrm{mot}}\left(Y, \overline{\mathbb{Q}_{\ell}}\right) \rightarrow D_{\mathrm{mot}}\left(X, \overline{\mathbb{Q}_{\ell}}\right), \\
f_{*}: D_{\mathrm{mot}}^{+}\left(X, \overline{\mathbb{Q}_{\ell}}\right) \rightarrow D_{\mathrm{mot}}^{+}\left(Y, \overline{\mathbb{Q}_{\ell}}\right), \quad f_{!}: D_{\mathrm{mot}}^{-}\left(X, \overline{\mathbb{Q}_{\ell}}\right) \rightarrow D_{\mathrm{mot}}^{-}\left(Y, \overline{\mathbb{Q}_{\ell}}\right) .
\end{gathered}
$$

If $f$ is relatively Deligne-Mumford, then we also have

$$
f_{*}: D_{\text {mot }}\left(X, \overline{\mathbb{Q}_{\ell}}\right) \rightarrow D_{\text {mot }}\left(Y, \overline{\mathbb{Q}_{\ell}}\right), \quad f_{!}: D_{\text {mot }}\left(X, \overline{\mathbb{Q}_{\ell}}\right) \rightarrow D_{\text {mot }}\left(Y, \overline{\mathbb{Q}_{\ell}}\right) .
$$

Proof. Note that $W(q)=R(q)^{\times} \cap M(q)$, where $R(q)$ is the integral closure of $\mathbb{Z}[1 / q]$ in $\overline{\mathbb{Q}}$. By [Z2, Variante 5.13, Section 6] (which extends easily to stacks not necessarily of separated diagonals), complexes with $R$-integral (resp. inverse $R$-integral) cohomology sheaves are preserved by the six operations and duality. By Remark 2.10, having Frobenius eigenvalues in $M\left(q^{n}\right)$ is equivalent to being mixed, and complexes with mixed cohomology sheaves are preserved by the operations by [S1, Remark 2.12].

As in [BBD, Stabilités 5.1.7], the theorem has the following consequence.

Corollary 2.14. The perverse truncation functors on $D\left(X, \overline{\mathbb{Q}_{\ell}}\right)$ preserve $D_{\text {mot }}\left(X, \overline{\mathbb{Q}_{\ell}}\right)$ and induce a t-structure on $D_{\text {mot }}\left(X, \overline{\mathbb{Q}_{\ell}}\right)$.

Theorems 0.1 (1) and 2.13 imply the following result on the structure of $D^{b}\left(X, \overline{\mathbb{Q}_{\ell}}\right)$. For $a \in \overline{\mathbb{Z}}_{\ell} \times$, we let $D_{\text {mot }}^{b}\left(X, \overline{\mathbb{Q}_{\ell}}\right)^{(a)} \subseteq D^{b}\left(X, \overline{\mathbb{Q}_{\ell}}\right)$ denote the full subcategory spanned by objects of the form $K^{(a)}$ with $K \in D_{\text {mot }}^{b}\left(X, \overline{\mathbb{Q}_{\ell}}\right)$. By definition, $D_{\text {mot }}^{b}\left(X, \overline{\mathbb{Q}_{\ell}}\right)^{(a)}$ only depends on the class of $a$ in $\overline{\mathbb{Z}}_{\ell} \times / W(q)$.

Theorem 2.15. For any stack $X$, we have a canonical decomposition for the bounded derived category of $\overline{\mathbb{Q}_{\ell}}$-sheaves:

$$
D^{b}\left(X, \overline{\mathbb{Q}_{\ell}}\right) \simeq \bigoplus_{a \in \overline{\mathbb{Z}}_{\ell} \times / W(q)} D_{\text {mot }}^{b}\left(X, \overline{\mathbb{Q}_{\ell}}\right)^{(a)}
$$


The case of schemes is [D4, Theorem B.7].

Proof. The proof is very similar to the case of schemes and parallel to the proof of Proposition 1.15. It suffices to show the following:

- (generation) Every object of $D^{b}\left(X, \overline{\mathbb{Q}_{\ell}}\right)$ is a successive extension of objects of $D_{\text {mot }}^{b}\left(X, \overline{\mathbb{Q}_{\ell}}\right)^{(a)}$;

- (orthogonality) $\operatorname{Hom}\left(A^{(a)}, B^{(b)}\right)=0$ for $A, B \in D_{\text {mot }}^{b}\left(X, \overline{\mathbb{Q}_{\ell}}\right), a / b \notin W(q)$.

The first point follows from Proposition 1.8 and Theorem 0.1 (1). For the orthogonality, note that

$$
\operatorname{Hom}\left(A^{(a)}, B^{(b)}\right) \simeq H^{0}\left(\operatorname{Spec}\left(\mathbb{F}_{q}\right), R \pi_{X *} R \mathcal{H} \text { om }(A, B)^{(b / a)}\right)=0,
$$

where $\pi_{X}: X \rightarrow \operatorname{Spec}\left(\mathbb{F}_{q}\right)$. Here we used the fact that $R \pi_{X *} R \mathcal{H}$ om $(A, B)$ is in $D_{\text {mot }}^{+}\left(\operatorname{Spec}\left(\mathbb{F}_{q}\right), \overline{\mathbb{Q}_{\ell}}\right)$ by Theorem 2.13 ,

Remark 2.16. The same decomposition holds for categories of $\overline{\mathbb{Q}_{\ell}}$-sheaves and perverse $\overline{\mathbb{Q}_{\ell}}$-sheaves. In particular, the subcategory of weakly motivic perverse sheaves $\operatorname{Perv}_{\text {mot }}\left(X, \overline{\mathbb{Q}_{\ell}}\right) \subseteq \operatorname{Perv}\left(X, \overline{\mathbb{Q}_{\ell}}\right)$ is stable under subquotient.

\section{Frobenius traces}

Theorem 0.1 (2) follows immediately from Theorem 0.1 (1) and the following.

Theorem 3.1. Let $X$ be a stack and let $\mathcal{F}$ be a Weil $\overline{\mathbb{Q}_{\ell}}$-sheaf on $X$. Then $E(\mathcal{F})$ is a finitely generated extension of $\mathbb{Q}$. In particular, $E(\mathcal{F})$ is a number field if and only if for all $n \geq 1$ and all $x \in X\left(\mathbb{F}_{q^{n}}\right)$, the eigenvalues of Frob ${ }_{x}$ acting on $\mathcal{F}_{\bar{x}}$ are algebraic numbers.

The case of schemes is a theorem of Deligne [D3, Théorème 3.1, Remarque 3.9].

Proof. To show the first assertion, by induction, we may replace $X$ by a dense open substack. In particular, we may assume that $\mathcal{F}$ is lisse. Moreover, by Lemma 1.11 and Remark 1.12 (or the fact that any sub-extension of a finitely generated field extension is finitely generated [B1, page V.113, Corollaire 3]), we may assume that $X$ is a DeligneMumford stack. We may further assume that $X \simeq[Y / G]$ for a finite group $G$ acting on an affine scheme $Y$. Choose an embedding $G \rightarrow \mathrm{GL}_{m}$ and take $Z=Y \wedge^{G} \mathrm{GL}_{m}=$ $\left(Y \times \mathrm{GL}_{m}\right) / G$. Then $f: Z \rightarrow X$ is a $\mathrm{GL}_{m}$-torsor. We have $E\left(f^{*} \mathcal{F}\right)=E(\mathcal{F})$. Indeed, any point $x \in X\left(\mathbb{F}_{q^{n}}\right)$ lifts to a point of $Z\left(\mathbb{F}_{q^{n}}\right)$ by Hilbert's Theorem 90 . We then apply the case of schemes [D3, Théorème 3.1, Remarque 3.9] to $f^{*} \mathcal{F}$ on $Z$. For the second assertion, it suffices to note that the Frobenius eigenvalues are algebraic numbers if and only if the Frobenius traces are algebraic numbers.

Corollary 3.2. Let $\mathcal{F}$ be a Weil $\overline{\mathbb{Q}_{\ell}}$-sheaf on a stack $X$ and let $\mathcal{G}$ be a subquotient of $\mathcal{F}$. Then $E(\mathcal{G})$ is contained in a finite extension of $E(\mathcal{F})$.

Proof. For each $x \in X\left(\mathbb{F}_{q^{n}}\right)$, the eigenvalues of $\operatorname{Frob}_{x}$ on $\mathcal{F}_{\bar{x}}$ are contained in a finite extension of $E\left(x^{*} \mathcal{F}\right) \subseteq E(\mathcal{F})$. The assertion then follows from the theorem, which says that $E(\mathcal{G})$ is generated by the traces $\operatorname{tr}\left(\operatorname{Frob}_{x}, \mathcal{G}_{\bar{x}}\right)$ at a finite number of points $x$ with varying $n$.

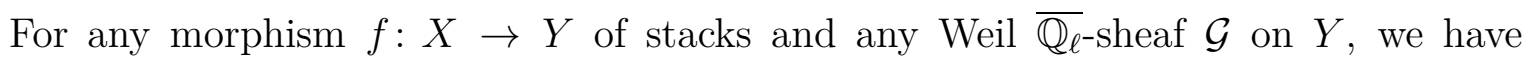
$E\left(f^{*} \mathcal{G}\right) \subseteq E(\mathcal{G})$ 
Corollary 3.3. Let $f: X \rightarrow Y$ be a morphism of stacks with $X$ nonempty and $Y$ connected. For any lisse Weil $\overline{\mathbb{Q}_{\ell}}$-sheaf $\mathcal{G}$ on $Y$, the field $E(\mathcal{G})$ is a finite extension of $E\left(f^{*} \mathcal{G}\right)$. In particular, for any $n \geq 1$ and any $y \in Y\left(\mathbb{F}_{q^{n}}\right)$, the field $E(\mathcal{G})$ is a finite extension of $E\left(y^{*} \mathcal{G}\right)$ (the field generated by $\operatorname{tr}\left(\operatorname{Frob}_{y}^{m}, \mathcal{F}_{\bar{y}}\right), m \geq 1$ ).

Proof. For any $y^{\prime} \in X\left(\mathbb{F}_{q^{n^{\prime}}}\right)$, the eigenvalues of Frob $y_{y^{\prime}}$ on $\mathcal{G}_{\overline{y^{\prime}}}$ are contained in a finite extension of $E\left(y^{*} \mathcal{G}\right)$ by Proposition 2.2. The second assertion then follows from the theorem, which says that $E(\mathcal{G})$ is generated by the traces $\operatorname{tr}\left(\right.$ Frob $\left._{y^{\prime}}, \mathcal{G}_{\overline{y^{\prime}}}\right)$ at a finite number of points $y^{\prime}$ with varying $n^{\prime}$. For the first assertion, let $x \in X\left(\mathbb{F}_{q^{n}}\right)$ and let $y=f(x)$. Then $E\left(y^{*} \mathcal{G}\right) \subseteq E\left(f^{*} \mathcal{G}\right) \subseteq E(\mathcal{G})$ and the first assertion follows from the second one.

Corollary 3.4. Let $f: X \rightarrow Y$ be a surjective morphism of stacks. Then, for any Weil $\overline{\mathbb{Q}_{\ell}}$-sheaf $\mathcal{G}$ on $Y$, the field $E(\mathcal{G})$ is a finite extension of $E\left(f^{*} \mathcal{G}\right)$.

Proof. This follows from Corollary 3.3 by taking a stratification of $Y$ by connected strata such that the restriction of $\mathcal{G}$ to each stratum is lisse.

Under additional assumptions, Corollary 3.3 admits the following refinement, which is a consequence of Gabber's theorem on the preservation of companionship.

Proposition 3.5. Let $f: X \rightarrow Y$ be a dominant open immersion of smooth stacks, $Y$ having separated diagonal, and let $\mathcal{G}$ be a lisse Weil $\overline{\mathbb{Q}_{\ell}}$-sheaf on $Y$. Then $E\left(f^{*} \mathcal{G}\right)=E(\mathcal{G})$. Moreover, if $\mathcal{G}^{\prime}$ is a lisse Weil $\overline{\mathbb{Q}_{\ell^{\prime}}}$-sheaf on $Y$ such that $f^{*} \mathcal{G}^{\prime}$ is a $\sigma$-companion of $f^{*} \mathcal{G}$ for some embedding $\sigma: E(\mathcal{G}) \rightarrow \overline{\mathbb{Q}_{\ell^{\prime}}}$, then $\mathcal{G}^{\prime}$ is a $\sigma$-companion of $\mathcal{G}$.

Proof. By the existence of smooth neighborhood [LMB, Théorème 6.3] (here we used the assumption that $Y$ has separated diagonal), any point $y \in Y\left(\mathbb{F}_{q^{n}}\right)$ factorizes through a smooth morphism $Y^{\prime} \rightarrow Y$, where $Y^{\prime}$ is a scheme. We are thus reduced to the case of schemes, which is a special case of [Z3, Proposition 3.10] (case $K$ a finite field and $G=\{1\})$, consequence of Gabber's theorem and purity.

As pointed out to us by Drinfeld, another way to prove the case of schemes of the proposition is to reduce by Drinfeld's version of Hilbert irreducibility [D4, Theorem 2.15] to the case of smooth curves, which is a theorem of Deligne [D1, Théorème 9.8].

\section{Companions}

In this section, we prove Theorem 0.2 on the existence of lisse companions on smooth stacks of separated diagonal and Corollary 0.3 on the existence of lisse companions on coarse moduli spaces. We then deduce the existence of perverse companions on stacks of separated diagonal (Theorem 4.14). We also deduce that companionship induces isomorphisms among the Grothendieck groups of Weil $\overline{\mathbb{Q}_{\ell}}$-sheaves for varying $\ell$ (Corollary 4.16).

To apply the reduction steps to Theorem 0.2 , again we need to show that we may shrink $X$. This is done by combining Proposition 3.5 with Drinfeld's theorem on the existence of companions on schemes. Let $E_{\lambda^{\prime}}$ be an algebraic extension of $\mathbb{Q}_{\ell^{\prime}}$.

Proposition 4.1. Let $j: U \rightarrow X$ be a dominant open immersion of smooth stacks, $X$ having separated diagonal. Let $\mathcal{F}$ be a lisse Weil $\overline{\mathbb{Q}_{\ell}}$-sheaf on $X$ and let $\sigma: E(\mathcal{F}) \rightarrow E_{\lambda^{\prime}}$ and $\iota^{\prime}: E_{\lambda^{\prime}} \rightarrow \mathbb{C}$ be embeddings. Assume that $j^{*} \mathcal{F}$ admits a lisse punctually $\iota^{\prime}$-pure $\sigma$ companion $\mathcal{G}^{\prime}$. Then $j_{*} \mathcal{G}^{\prime}$ is a lisse $\sigma$-companion of $\mathcal{F}$. 
Proof. If $j_{*} \mathcal{G}^{\prime}$ is lisse, then $j_{*} \mathcal{G}^{\prime}$ is a $\sigma$-companion of $\mathcal{F}$ by Proposition 3.5. It remains to show that $j_{*} \mathcal{G}^{\prime}$ is lisse. For this we may assume $E_{\lambda^{\prime}}=\overline{\mathbb{Q}_{\ell^{\prime}}}$. Since any pullback of $\mathcal{G}^{\prime}$ is punctually $\iota^{\prime}$-pure, we may assume that $X$ is a scheme. Since $\mathcal{G}^{\prime}$ is geometrically semisimple [D2, Théoème 3.4.1 (iii)], $\mathcal{G}_{\overline{\mathbb{F}_{q}}}^{\prime} \simeq\left(\mathcal{G}^{\prime \text { ss }}\right)_{\overline{\mathbb{F}_{q}}}$, where $\mathcal{G}^{\text {ss }}$ is the semisimplification of $\mathcal{G}^{\prime}$. Thus $j_{*} \mathcal{G}^{\prime}$ is lisse if and only if $j_{*} \mathcal{G}^{\prime \text { ss }}$ is lisse. By Drinfeld's theorem, $\mathcal{F}$ admits a lisse $\sigma$-companion $\mathcal{F}^{\prime}$, which we may assume semisimple. By Chebotarev's density theorem, we have $\mathcal{G}^{\prime \text { ss }} \simeq j^{*} \mathcal{F}^{\prime}$. Therefore, $j_{*} \mathcal{G}^{\text {sss }} \simeq j_{*} j^{*} \mathcal{F}^{\prime} \simeq \mathcal{F}^{\prime}$ is lisse.

Assuming Theorem 0.2 on the existence of lisse companions, we have the following consequence of Chebotarev's density theorem.

Proposition 4.2. Let $\mathcal{F}$ be a lisse Weil $\overline{\mathbb{Q}_{\ell}}$-sheaf on a geometrically unibranch stack $X$ and let $\sigma: E(\mathcal{F}) \rightarrow E_{\lambda^{\prime}}$ be an embedding. Then lisse $\sigma$-companions of $\mathcal{F}$ are unique up to semisimplification. Moreover, if $\mathcal{F}$ is simple, then, up to isomorphism, there exists at most one lisse $\sigma$-companion $\mathcal{F}^{\prime}$ of $\mathcal{F}$, and $\mathcal{F}^{\prime}$ is simple if it exists.

It is convenient to slightly extend terminology as follows. Given a Weil $\overline{\mathbb{Q}_{\ell}}$-sheaf $\mathcal{F}$ on a stack $X$ and an embedding $\sigma: E^{\prime} \rightarrow E_{\lambda^{\prime}}$ where $E^{\prime}$ is an extension of $E(\mathcal{F})$, we will refer to $(\sigma \mid E(\mathcal{F}))$-companions of $\mathcal{F}$ simply as $\sigma$-companions.

Proof. The first assertion follows from Chebotarev's density theorem (see Proposition 4.6 below). For the second assertion, it suffices to show the simplicity, as the uniqueness then follows from the first assertion. Up to replacing $X$ by a dense substack, we may assume that $X$ is smooth and of separated diagonal (Lemma 1.10). We may further assume that $E_{\lambda^{\prime}}=\overline{\mathbb{Q}_{\ell^{\prime}}}$. Extend $\sigma$ to an isomorphism $\overline{\mathbb{Q}_{\ell}} \stackrel{\sim}{\rightarrow} \overline{\mathbb{Q}_{\ell^{\prime}}}$, which we still denote by $\sigma$. Let $\mathcal{F}^{\prime}$ be a lisse $\sigma$-companion of $\mathcal{F}$. Up to replacing $\mathcal{F}^{\prime}$ by its semisimplification, we may assume $\mathcal{F}^{\prime} \simeq \bigoplus_{i} \mathcal{F}_{i}^{\prime}$, with each $\mathcal{F}_{i}^{\prime}$ simple. By Theorem 0.2 , there exists a $\sigma^{-1}$-companion (i.e. $\left(\sigma^{-1} \mid E\left(\mathcal{F}_{i}^{\prime}\right)\right)$-companion) $\mathcal{F}_{i}$ of $\mathcal{F}_{i}^{\prime}$. Then $\mathcal{F}$ is the semisimplification of $\bigoplus_{i} \mathcal{F}_{i}$, since they are both $\sigma^{-1}$-companions of $\mathcal{F}^{\prime}$. Thus $\mathcal{F}_{i}=0$ for all but one $i$, and the same holds for $\mathcal{F}_{i}^{\prime}$. Therefore, $\mathcal{F}^{\prime}$ is simple.

Proof of Theorem 0.2. By Corollary 3.2, we may assume $X$ irreducible and $\mathcal{F}$ simple. In this case, we show in addition to the statements of the theorem, that any lisse $\sigma$ companion $\mathcal{F}^{\prime}$ is punctually $\iota^{\prime}$-pure for any embedding $\iota^{\prime}: \overline{\mathbb{Q}_{\ell^{\prime}}} \rightarrow \mathbb{C}$. By Proposition 4.1 and Proposition 3.5 (or Corollary [3.3), we may shrink $X$. Applying Lemma 1.11 and Remark 1.12 (or Corollary [3.4), we reduce to the case where $X$ is a Deligne-Mumford stack. Up to shrinking $X$, we may further assume that $X=[Y / G]$, where $G$ is a finite group acting on an affine scheme $Y$. Choose an embedding $G \rightarrow \mathrm{GL}_{m}$. Consider the embedding $\mathrm{GL}_{m} \rightarrow \mathbb{A}^{m^{2}}$, which is equivariant under the action of $\mathrm{GL}_{m}$. Let $p: Z=$ $\left[Y \times \mathbb{A}^{m^{2}} / G\right] \rightarrow[Y / G]$ be the projection and let $s$ be the zero section. Note that $Z$ is smooth. Since $s^{*} p^{*} \mathcal{F} \simeq \mathcal{F}, p^{*} \mathcal{F}$ is simple. It suffices to show the assertions for $\left(Z, p^{*} \mathcal{F}\right)$. Indeed, if $\mathcal{G}^{\prime}$ is a lisse $\sigma$-companion of $p^{*} \mathcal{F}$, then $\mathcal{F}^{\prime}=s^{*} \mathcal{G}^{\prime}$ is a $\sigma$-companion of $s^{*} p^{*} \mathcal{F} \simeq \mathcal{F}$. Applying Propositions 4.1 and 3.5 to the dense open subscheme $\left[Y \times \mathrm{GL}_{m} / G\right]$ of $Z$, we are reduced to the case of schemes. In this case, the existence of lisse companions follows from Drinfeld's theorem ([D4, Theorem 1.1, Section 1.2] applied to a twist $\mathcal{F}^{(a)}$ of $\mathcal{F}$ such that $\operatorname{det}\left(\mathcal{F}^{(a)}\right)$ has finite order). Moreover, if $\mathcal{F}^{\prime}$ is a lisse $\sigma$-companion of $\mathcal{F}$, then $\mathcal{F}^{\prime}$ is simple by Proposition 4.2 (which can be applied, because Proposition 4.2 for schemes depends only on the known existence of lisse companions on smooth schemes), hence punctually $\iota$-pure. 
Definition 4.3. Let $f: X \rightarrow Y$ be a morphism of stacks. We say that $f$ creates lisse companions if for every lisse Weil $\overline{\mathbb{Q}_{\ell}}$-sheaf $\mathcal{G}$ on $Y$ and every embedding $\sigma: E(\mathcal{G}) \rightarrow E_{\lambda^{\prime}}$ such that $f^{*} \mathcal{G}$ admits a $\sigma$-companion, $\mathcal{G}$ admits a $\sigma$-companion.

Note that we do not ask for the existence of a companion $\mathcal{G}^{\prime}$ of $\mathcal{G}$ such that $f^{*} \mathcal{G}^{\prime}$ is isomorphic to a given companion of $f^{*} \mathcal{G}$. Morphisms creating lisse companions are stable under composition. If $X \stackrel{f}{\rightarrow} Y \stackrel{g}{\rightarrow} Z$ is a sequence of morphisms of stacks, and if $g f$ creates lisse companions, then $g$ creates lisse companions.

Proposition 4.4. Let $f: X \rightarrow Y$ be a morphism of stacks. Then $f$ creates lisse companions if it satisfies any of the following conditions:

(1) $f$ is a proper universal homeomorphism.

(2) $f: X=\coprod_{i} X_{i} \rightarrow Y$, where $\left(X_{i}\right)$ is a finite Zariski open cover and $Y$ is geometrically unibranch.

Proof. Let $\mathcal{G}$ be a lisse Weil $\overline{\mathbb{Q}_{\ell}}$-sheaf on $Y$, let $\sigma: E(\mathcal{G}) \rightarrow E_{\lambda^{\prime}}$ be an embedding, and let $\mathcal{F}^{\prime}$ be a lisse $\sigma$-companion of $f^{*} \mathcal{G}$.

(1) Let us first note that for any proper morphism $f$ of stacks with geometrically connected fibers, the adjunction map $a: \mathcal{G} \rightarrow f_{*} f^{*} \mathcal{G}$ is an isomorphism and the adjunction map $b: f^{*} f_{*} \mathcal{F}^{\prime} \rightarrow \mathcal{F}^{\prime}$ is a monomorphism. Indeed, by proper base change, the stalk of $a$ at any geometric point $\bar{y} \rightarrow Y$ can be identified with the isomorphism $\mathcal{G}_{\bar{y}} \rightarrow \Gamma\left(X_{\bar{y}}, \mathcal{G}_{\bar{y}}\right)$, and the stalk of $b$ at a geometric point $\bar{x} \rightarrow X$ above $\bar{y} \rightarrow Y$ can be identified with the injection $\Gamma\left(X_{\bar{y}}, \mathcal{F}^{\prime} \mid X_{\bar{y}^{\prime}}\right) \rightarrow \mathcal{F}_{\bar{x}}^{\prime}$. Now let $f$ be a proper universal homeomorphism. By Lemma 4.5 below, $f_{*} \mathcal{F}^{\prime}$ is a $\sigma$-companion of $f_{*} f^{*} \mathcal{G} \simeq \mathcal{G}$. It remains to show that $f_{*} \mathcal{F}^{\prime}$ is lisse. We have seen that $b$ is a monomorphism. Both $f^{*} f_{*} \mathcal{F}^{\prime}$ and $\mathcal{F}^{\prime}$ are $\sigma$-companions of $f^{*} \mathcal{G}$. In other words, for any $x \in X\left(\mathbb{F}_{q^{n}}\right)$, the characteristic polynomials of Frob $_{y}$ acting on $\left(f^{*} f_{*} \mathcal{F}^{\prime}\right)_{\bar{x}}$ and $\mathcal{F}_{\bar{x}}^{\prime}$ coincide. In particular, $\left(f^{*} f_{*} \mathcal{F}^{\prime}\right)_{\bar{x}}$ and $\mathcal{F}_{\bar{x}}^{\prime}$ have the same rank. Thus, $b$ is an isomorphism. By Lemma [1.4, $f_{*} \mathcal{F}^{\prime}$ is lisse.

(2) We may assume $Y$ irreducible and each $X_{i}$ nonempty. Then $U=\bigcap_{i} X_{i}$ is nonempty. Let $j: U \rightarrow Y$ and let $\mathcal{G}_{U}^{\prime}$ be a semisimple lisse $\sigma$-companion of $j^{*} \mathcal{G}$. Let $\mathcal{F}_{i}^{\prime}=\mathcal{F}^{\prime} \mid X_{i}$. Then $\mathcal{G}_{U}^{\prime} \simeq \mathcal{F}_{i}^{\text {sss }} \mid U$, so that $j_{*} \mathcal{G}_{U}^{\prime} \mid X_{i} \simeq \mathcal{F}_{i}^{\text {sss }}$. Thus $j_{*} \mathcal{G}_{U}^{\prime}$ is a lisse $\sigma$-companion of $\mathcal{G}$.

Lemma 4.5. Let $f: X \rightarrow Y$ be a proper universal homeomorphism of stacks. Let $\mathcal{F}$ be a $\overline{\mathbb{Q}_{\ell}}$-sheaf on $X$ and let $\mathcal{F}^{\prime}$ be a $\sigma$-companion of $\mathcal{F}$, where $\sigma: E(\mathcal{F}) \rightarrow E_{\lambda^{\prime}}$ is an embedding. Then $E\left(f_{*} \mathcal{F}\right) \subseteq E(\mathcal{F})$ and $f_{*} \mathcal{F}^{\prime}$ is a $\sigma$-companion of $f_{*} \mathcal{F}$.

Proof. By proper base change, we may assume that $Y=\operatorname{Spec}\left(\mathbb{F}_{q^{n}}\right)$ is a point. We may further assume that $X$ is reduced. In this case, $X=B G$ for a group scheme $G$ over $Y$. Applying the proof of Lemma 1.11, we get $f=h g$, where $B G \stackrel{g}{\rightarrow} B\left(G / G^{0}\right) \stackrel{h}{\rightarrow} Y$. We have $\mathcal{F} \simeq g^{*} g_{*} \mathcal{F}$ and $\mathcal{F}^{\prime} \simeq g^{*} g_{*} \mathcal{F}^{\prime}$. By Remark 1.12, $E\left(g_{*} \mathcal{F}\right)=E(\mathcal{F})$ and $g_{*} \mathcal{F}^{\prime}$ is a $\sigma$-companion of $g_{*} \mathcal{F}$. We are thus reduced to showing that $E\left(h_{*}-\right) \subseteq E(-)$ and that $h_{*}$ preserves $\sigma$-companions. For this case, we apply [Z3, Proposition 5.8] recalled as part of Theorem 4.11 below (or the trace formula [S1, Theorem 4.2]).

Proof of Corollary 0.3. By Proposition 4.4 (2), we may assume that $X$ is the coarse moduli space of a smooth stack $Y$ with finite inertia. It then suffices to apply Proposition 4.4 (1) to the proper universal homeomorphism $f: Y \rightarrow X$ and Theorem 0.2 to $Y$. 
In the rest of the section, we discuss companions of perverse sheaves and in Grothendieck groups. For this, it is convenient to introduce perverse Weil sheaves. Let $E_{\lambda}$ be an algebraic extension of $\mathbb{Q}_{\ell}$. A perverse Weil $E_{\lambda}$-sheaf on a stack $X$ is a perverse $E_{\lambda}$-sheaf $\mathcal{P}$ on $X \otimes_{\mathbb{F}_{q}} \overline{\mathbb{F}_{q}}$ equipped with an action of the Weil group $W\left(\overline{\mathbb{F}_{q}} / \mathbb{F}_{q}\right)$ lifting the action of $W\left(\overline{\mathbb{F}_{q}} / \mathbb{F}_{q}\right)$ on $X \otimes_{\mathbb{F}_{q}} \overline{\mathbb{F}_{q}}$. A morphism of perverse Weil $E_{\lambda}$-sheaves on $X$ is a morphism of the underlying perverse $E_{\lambda}$-sheaves on $X \otimes_{\mathbb{F}_{q}} \overline{\mathbb{F}_{q}}$ compatible with the action of $W\left(\overline{\mathbb{F}_{q}} / \mathbb{F}_{q}\right)$. As in the case of schemes [BBD, Proposition 5.1.2] or $E_{\lambda}$-sheaves (Remark 1.2), we have a fully faithful functor $\operatorname{Perv}^{W}\left(X, E_{\lambda}\right) \rightarrow \operatorname{Perv}\left(X, E_{\lambda}\right)$ and the essential image is stable under extension. Remark [1.6 on extending scalars to $\overline{\mathbb{Q}_{\ell}}$ still holds. The analogue of Proposition 1.15 holds with the same proof:

$$
\operatorname{Perv}^{W}\left(X, \overline{\mathbb{Q}_{\ell}}\right) \simeq \underset{a \in \overline{\mathbb{Q}}^{\times} / \overline{\mathbb{Z}}_{\ell} \times}{ } \operatorname{Perv}\left(X, \overline{\mathbb{Q}_{\ell}}\right)^{(a)} .
$$

We let $K_{\text {lisse }}^{W}\left(X, E_{\lambda}\right)$ denote the Grothendieck group of $\operatorname{Shv}_{\text {lisse }}^{W}\left(X, E_{\lambda}\right)$, which is a free Abelian group generated by the isomorphism classes of simple lisse Weil $E_{\lambda}$-sheaves on $X$. We let $K^{W}\left(X, E_{\lambda}\right)$ denote the Grothendieck group of $\operatorname{Shv}^{W}\left(X, E_{\lambda}\right)$, which is also the Grothendieck group of $\operatorname{Perv}^{W}\left(X, E_{\lambda}\right)$, and is a free Abelian group generated by the isomorphism classes of simple perverse Weil $E_{\lambda}$-sheaves on $X$. For a Weil sheaf or Weil perverse sheaf $\mathcal{F}$, we let $[\mathcal{F}]$ denote its class in the Grothendieck group. We have a commutative diagram

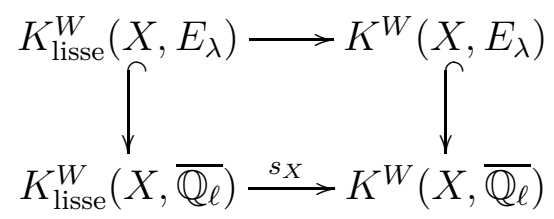

of Abelian groups. That the vertical arrows are injections is standard (cf. [B2, page VIII.191, Théorème 1]).

We have the following Chebotarev's density theorem, generalizing [SZ, Lemma 4.1.4].

Proposition 4.6. Let $X$ be a stack.

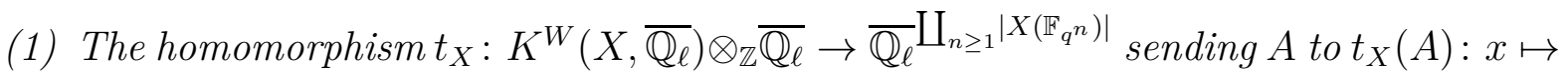
$\operatorname{tr}\left(\right.$ Frob $\left._{x}, A_{\bar{x}}\right)$ is injective.

(2) For $X$ irreducible and geometrically unibranch, the homomorphism $s_{X}: K_{\text {lisse }}^{W}\left(X, \overline{\mathbb{Q}_{\ell}}\right) \rightarrow$ $K^{W}\left(X, \overline{\mathbb{Q}_{\ell}}\right)$ is injective and the conjugates of the images of Frob ${ }_{x}$ in the fundamental group $\pi_{1}(X)$ form a dense subset.

Here as before $\left|X\left(\mathbb{F}_{q^{n}}\right)\right|$ denotes the set of isomorphism classes of the groupoid $X\left(\mathbb{F}_{q^{n}}\right)$. The Frobenius traces are extended to $K^{W}\left(X, \overline{\mathbb{Q}_{\ell}}\right) \otimes_{\mathbb{Z}} \overline{\mathbb{Q}_{\ell}}$ by linearity: for $A=\sum_{i} c_{i}\left[\mathcal{F}_{i}\right]$ with $c_{i} \in \overline{\mathbb{Q}_{\ell}}, \operatorname{tr}\left(\operatorname{Frob}_{x}, A_{\bar{x}}\right)=\sum_{i} c_{i} \operatorname{tr}\left(\operatorname{Frob}_{x},\left(\mathcal{F}_{i}\right)_{\bar{x}}\right)$.

Proof. We extend cohomological operations to $K^{W}\left(X, \overline{\mathbb{Q}_{\ell}}\right) \otimes_{\mathbb{Z}} \overline{\mathbb{Q}_{\ell}}$ by linearity (cf. Remark 4.9 below). Let $A \in \operatorname{Ker}\left(t_{X}\right)$. There exists a stratification of $X$ by geometrically unibranch substacks $j_{\alpha}: X_{\alpha} \rightarrow X$ such that for each $\alpha, j_{\alpha}^{*} A$ belongs to the image of $s_{X_{\alpha}} \otimes \overline{\mathbb{Q}_{\ell}}$. We have $t_{X_{\alpha}}\left(j_{\alpha}^{*} A\right)=0$ and $A=\sum_{\alpha} j_{\alpha} ! j_{\alpha}^{*} A$ and for (1) it suffices to show $j_{\alpha}^{*} A=0$. Changing notation, it suffices to show that for $X$ irreducible and geometrically unibranch, $t_{X}\left(s_{X} \otimes \overline{\mathbb{Q}_{\ell}}\right)$ is an injection. Note that this implies the injectivity of $s_{X}$. (Alternatively we can apply the reduction in the proof of [L3, Théorème 1.1.2].) 
For $x \in X\left(\mathbb{F}_{q^{n}}\right)$ and $A \in K\left(X, \overline{\mathbb{Q}}_{\ell}\right)^{(a)}, a \in \overline{\mathbb{Q}}_{\ell} \times$, the reciprocal zeroes and roots of $\operatorname{det}\left(1-t\right.$ Frob $\left._{x}, A_{\bar{x}}\right)$ belong to $a^{n} \overline{\mathbb{Z}}_{\ell} \times$. It follows that

$$
t_{X}\left(K^{W}\left(X, \overline{\mathbb{Q}_{\ell}}\right) \otimes_{\mathbb{Z}} \overline{\mathbb{Q}_{\ell}}\right)=\bigoplus_{a \in \overline{\mathbb{Q}}_{\ell} \times / \overline{\mathbb{Z}}_{\ell} \times} t_{X}\left(K\left(X, \overline{\mathbb{Q}_{\ell}}\right)^{(a)} \otimes_{\mathbb{Z}} \overline{\mathbb{Q}_{\ell}}\right) .
$$

Thus it suffices to show that $t_{X}^{\text {lisse }}:=t_{X}\left(s_{X} \otimes \overline{\mathbb{Q}_{\ell}}\right)$ is an injection on $K\left(X, \overline{\mathbb{Q}_{\ell}}\right) \otimes_{\mathbb{Z}} \overline{\mathbb{Q}_{\ell}}$. This is equivalent to the density of the conjugates of Frob $_{x}$ in the fundamental group $\pi_{1}(X)$ [CR, Corollary 27.13].

Since for any nonempty open substack $U$ of $X, \pi_{1}(U) \rightarrow \pi_{1}(X)$ is a surjection, we may shrink $X$. We reduce to the case of Deligne-Mumford stacks as follows. Let $A=\sum_{i} c_{i}\left[\mathcal{F}_{i}\right]$ be an element in the kernel of $t_{X}^{\text {lisse }}$ with $\mathcal{F}_{i} \in \operatorname{Shv}_{\text {lisse }}\left(X, \overline{\mathbb{Q}_{\ell}}\right)$ and $c_{i} \in \overline{\mathbb{Q}_{\ell}}$. We apply Lemma 1.11 to $\bigoplus_{i} \mathcal{F}_{i}$ to find, up to shrinking $X$, a gerbe-like morphism $f: X \rightarrow Y$ where $Y$ is a Deligne-Mumford stack such that $f^{*} f_{*} \mathcal{F}_{i} \simeq \mathcal{F}_{i}$ for all $i$. By Remark 1.12, $t_{Y}^{\text {lisse }}\left(f_{*} A\right)=0$, where $f_{*} A=\sum_{i} c_{i}\left[f_{*} \mathcal{F}_{i}\right]$. Thus, by Chebotarev's density theorem for Deligne-Mumford stacks [SZ, Lemmas 4.1.4, 4.1.5], we have $f_{*} A=0$. Therefore, $A=f^{*} f_{*} A=0$.

The definitions of $E(\mathcal{F})$ and $\sigma$-companions at the beginning of the paper extend to elements of Grothendieck groups and to perverse Weil sheaves as follows. Given $A \in$ $K^{W}\left(X, \overline{\mathbb{Q}_{\ell}}\right)$, we let $E(A)$ denote the subfield of $\overline{\mathbb{Q}_{\ell}}$ generated by $\operatorname{tr}\left(\operatorname{Frob}_{x}, A_{\bar{x}}\right)$, where $x \in X\left(\mathbb{F}_{q^{n}}\right)$ and $n \geq 1$. Let $\sigma: E \rightarrow E_{\lambda^{\prime}}$ be a field embedding, where $E$ is an extension of $E(A)$. We say that $A^{\prime} \in K^{W}\left(X, E_{\lambda^{\prime}}\right)$ is a $\sigma$-companion of $A$ if for all $x \in X\left(\mathbb{F}_{q^{n}}\right)$ with $n \geq 1$, we have $\operatorname{tr}\left(\operatorname{Frob}_{x}, A_{\bar{x}}^{\prime}\right)=\sigma \operatorname{tr}\left(\operatorname{Frob}_{x}, A_{\bar{x}}\right)$. For a perverse Weil $\overline{\mathbb{Q}}_{\ell}$-sheaf $\mathcal{P}$, we write $E(\mathcal{P})=E([\mathcal{P}])$. By a perverse $\sigma$-companion of $\mathcal{P}$, we mean a perverse Weil

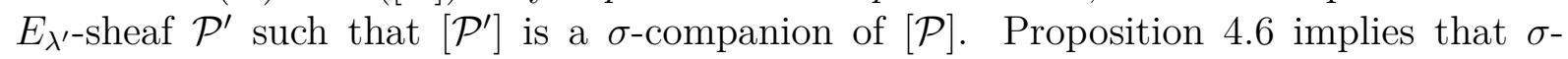
companions in Grothendieck groups are unique and perverse $\sigma$-companions are unique up to semisimplification.

Let $K_{\text {mot }}\left(X, \overline{\mathbb{Q}_{\ell}}\right)$ denote the Grothendieck group of $\operatorname{Perv}_{\text {mot }}\left(X, \overline{\mathbb{Q}_{\ell}}\right)$ (see Remark 2.16), which is also the Grothendieck group of $D_{\text {mot }}^{b}\left(X, \overline{\mathbb{Q}_{\ell}}\right)$. Then we have

$$
K^{W}\left(X, \overline{\mathbb{Q}_{\ell}}\right) \simeq \bigoplus_{a \in \overline{\mathbb{Q}}_{\ell}^{\times} / W(q)} K_{\mathrm{mot}}\left(X, \overline{\mathbb{Q}_{\ell}}\right)^{(a)}
$$

\section{Remark 4.7.}

(1) Let $A \in K^{W}\left(X, \overline{\mathbb{Q}_{\ell}}\right)$ and $A^{\prime} \in K^{W}\left(X, \overline{\mathbb{Q}_{\ell^{\prime}}}\right)$. Let $E \subseteq \overline{\mathbb{Q}_{\ell}}$ and let $\sigma: E \rightarrow \overline{\mathbb{Q}_{\ell^{\prime}}}$ be an embedding. If for every embedding $\tau: \overline{\mathbb{Q}_{\ell}} \rightarrow \overline{\mathbb{Q}_{\ell^{\prime}}}$ extending $\sigma, A^{\prime}$ is a $\tau$-companion of $A$, then $E(A) \subseteq E$. Indeed, if $t \in \overline{\mathbb{Q}_{\ell}}$ and $t^{\prime} \in \overline{\mathbb{Q}_{\ell^{\prime}}}$ are such that for every $\tau$ extending $\sigma$, we have $\tau(t)=t^{\prime}$, then $t \in E$.

(2) Let $\pi_{a}: K^{W}\left(X, \overline{\mathbb{Q}_{\ell}}\right) \rightarrow K_{\text {mot }}\left(X, \overline{\mathbb{Q}_{\ell}}\right)^{(a)}$ be the projection and let $\tau: \overline{\mathbb{Q}_{\ell}} \rightarrow \overline{\mathbb{Q}_{\ell^{\prime}}}$ be an embedding. If $A^{\prime}$ is a $\tau$-companion of $A \in K^{W}\left(X, \overline{\mathbb{Q}_{\ell}}\right)$, then $\pi_{\tau a} A^{\prime}$ is a $\tau$ companion of $\pi_{a} A$. Indeed, for $x \in X\left(\mathbb{F}_{q^{n}}\right)$, the set of reciprocal zeroes and roots of $\operatorname{det}\left(1-t \operatorname{Frob}_{x},\left(\pi_{a} A\right)_{\bar{x}}\right)$ is the intersection of $a^{n} W\left(q^{n}\right)$ and the set of reciprocal zeroes and roots of $\operatorname{det}\left(1-t \operatorname{Frob}_{x}, A_{\bar{x}}\right)$, with the same multiplicities: if $\operatorname{tr}\left(\operatorname{Frob}_{x}, A_{\bar{x}}\right)=$ $\sum_{\lambda} m_{\lambda} \lambda$, then $\operatorname{tr}\left(\operatorname{Frob}_{x},\left(\pi_{a} A\right)_{\bar{x}}\right)=\sum_{\lambda \in a^{n} W\left(q^{n}\right)} m_{\lambda} \lambda$, so that

$$
\tau \operatorname{tr}\left(\operatorname{Frob}_{x},\left(\pi_{a} A\right)_{\bar{x}}\right)=\sum_{\lambda \in a^{n} W\left(q^{n}\right)} m_{\lambda} \tau \lambda=\operatorname{tr}\left(\operatorname{Frob}_{x},\left(\pi_{\tau a} A^{\prime}\right)_{\bar{x}}\right) .
$$

Proposition 4.8. Let $X$ be a stack and let $A \in K^{W}\left(X, \overline{\mathbb{Q}_{\ell}}\right)$. The following conditions are equivalent: 
(1) $E(A)$ is a number field.

(2) $\operatorname{tr}\left(\operatorname{Frob}_{x}, A_{\bar{x}}\right)$ is an algebraic number for all $n \geq 1$ and all $x \in X\left(\mathbb{F}_{q^{n}}\right)$.

(3) A belongs to $K_{\mathrm{alg}}^{W}\left(X, \overline{\mathbb{Q}_{\ell}}\right):=\bigoplus_{a \in \overline{\mathbb{Q}}^{\times} / W(q)} K_{\operatorname{mot}}\left(X, \overline{\mathbb{Q}_{\ell}}\right)^{(a)}$.

Thus, if we identify $K^{W}\left(X, \overline{\mathbb{Q}_{\ell}}\right)$ with its image under $t_{X}$, then

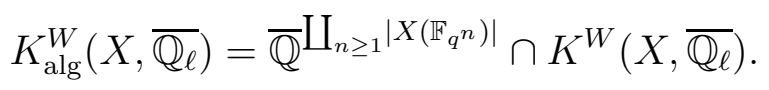

Proof. It is clear that (1) implies (2). By Theorem 3.1, (3) implies (1). Now assume that (2) holds. Let $A=B+C$, where $B$ is the projection of $A$ in $K_{\mathrm{alg}}^{W}\left(X, \overline{\mathbb{Q}_{\ell}}\right)$. Since $\operatorname{det}\left(1-t \operatorname{Frob}_{x}, A_{\bar{x}}\right) \in \overline{\mathbb{Q}}(t)$, we have $\operatorname{det}\left(1-t \operatorname{Frob}_{x}, C_{\bar{x}}\right)=1$, so that $\operatorname{tr}\left(\operatorname{Frob}_{x}^{m}, C_{\bar{x}}\right)=0$ for $m \geq 1$. Thus $C=0$ by Proposition 4.6.

For $w \in \mathbb{Z}$, let $K_{w}^{W}\left(X, \overline{\mathbb{Q}_{\ell}}\right)$ denote the Grothendieck group of perverse Weil $\overline{\mathbb{Q}_{\ell}}$-sheaves pure of weight $w$. We have, by Remark 2.10 ,

$$
K_{\mathrm{m}}^{W}\left(X, \overline{\mathbb{Q}_{\ell}}\right):=\bigoplus_{a \in M(q) / W(q)} K_{\mathrm{mot}}\left(X, \overline{\mathbb{Q}_{\ell}}\right)^{(a)}=\bigoplus_{w \in \mathbb{Z}} K_{w}^{W}\left(X, \overline{\mathbb{Q}_{\ell}}\right) .
$$

Remark 4.9. Let $f: X \rightarrow Y$ be a morphism of stacks, we have (bi)linear maps

$$
\begin{gathered}
p_{w}: K_{\mathrm{m}}^{W}\left(X, \overline{\mathbb{Q}_{\ell}}\right) \rightarrow K_{w}^{W}\left(X, \overline{\mathbb{Q}_{\ell}}\right), \\
-\otimes-, \overline{H o m}(-,-): K^{W}\left(X, \overline{\mathbb{Q}_{\ell}}\right) \times K^{W}\left(X, \overline{\mathbb{Q}_{\ell}}\right) \rightarrow K^{W}\left(X, \overline{\mathbb{Q}_{\ell}}\right), \\
D_{X}: K^{W}\left(X, \overline{\mathbb{Q}_{\ell}}\right) \rightarrow K^{W}\left(X, \overline{\mathbb{Q}_{\ell}}\right), \\
f^{*}, f^{!}: K^{W}\left(Y, \overline{\mathbb{Q}_{\ell}}\right) \rightarrow K^{W}\left(X, \overline{\mathbb{Q}_{\ell}}\right),
\end{gathered}
$$

where $p_{w}$ is the projection, $w \in \mathbb{Z}$. If $f$ is relatively Deligne-Mumford, then we have linear maps

$$
f_{*}, f_{!}: K^{W}\left(X, \overline{\mathbb{Q}_{\ell}}\right) \rightarrow K^{W}\left(Y, \overline{\mathbb{Q}_{\ell}}\right) .
$$

For an immersion of stacks $f: X \rightarrow Y$, the middle extension functor $f_{! *}: \operatorname{Perv}^{W}\left(X, \overline{\mathbb{Q}_{\ell}}\right) \rightarrow$ $\operatorname{Perv}^{W}\left(Y, \overline{\mathbb{Q}_{\ell}}\right)$ is not exact in general. We define a linear map

$$
f_{!_{*}}: K^{W}\left(X, \overline{\mathbb{Q}_{\ell}}\right) \rightarrow K^{W}\left(Y, \overline{\mathbb{Q}_{\ell}}\right)
$$

such that $f_{! *}[\mathcal{P}]=\left[f_{! *} \mathcal{P}\right]$ for $\mathcal{P} \in \operatorname{Perv}^{W}\left(X, \overline{\mathbb{Q}_{\ell}}\right)$ semisimple.

The definition of $f_{! *}$ on Grothendieck groups is further justified by the following fact. Let $\iota: \overline{\mathbb{Q}_{\ell}} \rightarrow \mathbb{C}$ be an embedding and let $w \in \mathbb{R}$. We let $\operatorname{Perv}_{\iota,\{w, w+1\}}^{W}\left(X, \overline{\mathbb{Q}_{\ell}}\right)$ denote the category of perverse Weil $\overline{\mathbb{Q}_{\ell}}$-sheaves on $X, \iota$-mixed of weights $w$ and $w+1$. Then $f_{! *}[\mathcal{P}]=\left[f_{! *} \mathcal{P}\right]$ for $\mathcal{P} \in \operatorname{Perv}_{\iota,\{w, w+1\}}^{W}\left(X, \overline{\mathbb{Q}_{\ell}}\right)$ by the following immediate extension from the case of schemes ([Z1, Corollaire 2.10], [SZ, Lemma 4.1.8]).

Lemma 4.10. Let $f: X \rightarrow Y$ be an immersion of stacks. The functor

$$
f_{! *}: \operatorname{Perv}_{\iota,\{w, w+1\}}^{W}\left(X, \overline{\mathbb{Q}_{\ell}}\right) \rightarrow \operatorname{Perv}_{\iota,\{w, w+1\}}^{W}\left(Y, \overline{\mathbb{Q}_{\ell}}\right)
$$

is exact.

We have the following generalization of theorems of Gabber. 
Theorem 4.11. Let $X$ and $Y$ be stacks with separated diagonal. Then the operations in Remark 4.9 preserve $E$ and $\sigma$-companions. More precisely, for any operation $F$ in the list and $A \in K^{W}\left(X, \overline{\mathbb{Q}_{\ell}}\right), E(F A) \subseteq E(A)$, and for any $\sigma: E(A) \rightarrow \overline{\mathbb{Q}_{\ell^{\prime}}}$ and any $\sigma$-companion $A^{\prime}$ of $A, F A^{\prime}$ is a $\sigma$-companion of $F A$.

By biduality $D_{X} D_{X} A=A$, it follows that $E\left(D_{X} A\right)=E(A)$.

Proof. The assertion on the six operations and duality is the case over a finite field of [Z3, Proposition 5.8] (extended to Weil sheaves by Remark 4.7] or [Z3, Remarque 4.16]), generalizing a theorem of Gabber [F, Theorem 2]. For the assertion on $p_{w}$ and $f_{! *}$, where $f$ is an open immersion, we reduce to the case of separated schemes by the existence of smooth neighborhoods [LMB, Théorème 6.3]. The assertion on $p_{w}$ is then [Z3, Proposition 2.7] (again extended to Weil sheaves), a consequence of Gabber's theorem on $f_{\text {!* }}$ on pure perverse sheaves. For $f_{! *}$, by Remark 4.7(1), it suffices to show that $f_{! *}$ on $K^{W}$ preserves $\tau$ companions, where $\tau: \overline{\mathbb{Q}_{\ell}} \rightarrow \overline{\mathbb{Q}_{\ell^{\prime}}}$ is an embedding extending $\sigma$. Let $A^{\prime} \in K^{W}\left(X, \overline{\mathbb{Q}_{\ell^{\prime}}}\right)$ be a

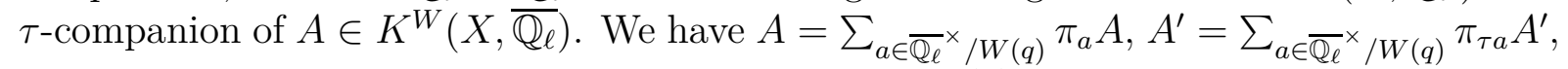
and $\pi_{\tau a} A^{\prime}$ is a $\tau$-companion of $\pi_{a} A$ by Remark 4.7 (2). Thus, up to replacing $A$ by $\left(\pi_{a} A\right)^{\left(1 / a_{0}\right)}$ and $A^{\prime}$ by $\left(\pi_{\tau a} A^{\prime}\right)^{\left(1 / \tau a_{0}\right)}$, where $a_{0} \in \overline{\mathbb{Q}}_{\ell} \times$ is a representative of $a$, we may assume $A, A^{\prime} \in K_{\text {mot }} \subseteq K_{\mathrm{m}}^{W}$. Similarly, since $A=\sum_{w \in \mathbb{Z}} p_{w} A, A^{\prime}=\sum_{w \in \mathbb{Z}} p_{w} A^{\prime}$, and $p_{w}$ preserves $\tau$-companions, up to replacing $A$ by $p_{w} A$ and $A^{\prime}$ by $p_{w} A^{\prime}$, we are reduced to the case when $A, A^{\prime} \in K$ are pure of weight $w$, which is Gabber's theorem [F, Theorem 3].

Due to cancellation in the alternating sum, the analogues of Corollaries 3.3 and 3.4 do not hold: $E(A)$ is not necessarily a finite extension of $E\left(f^{*} A\right)$ for $f: X \rightarrow Y$ surjective even for $A \in K_{\text {lisse }}^{W}\left(Y, \overline{\mathbb{Q}_{\ell}}\right)$. For example, for $f: \operatorname{Spec}\left(\mathbb{F}_{q^{2}}\right) \rightarrow \operatorname{Spec}\left(\mathbb{F}_{q}\right)$ and $A=\left[\overline{\mathbb{Q}}_{\ell}^{(a)}\right]-$ $\left[\overline{\mathbb{Q}}^{(-a)}\right]$, we have $E(A)=\mathbb{Q}(a)$ but $E\left(f^{*} A\right)=\mathbb{Q}$.

The analogue of Proposition 3.5 holds for $K_{\text {lisse }}^{W}$ with the same proof. Moreover, Corollary 3.3 has the following refinement under additional assumptions.

Proposition 4.12. Let $f: X \rightarrow Y$ be a morphism of stacks of irreducible geometric fibers. Let $A \in K^{W}\left(Y, \overline{\mathbb{Q}_{\ell}}\right)$. Then $E\left(f^{*} A\right)=E(A)$. Moreover, if $A^{\prime}$ is a Weil $\overline{\mathbb{Q}_{\ell^{\prime}}}$-sheaf on $Y$ such that $f^{*} A^{\prime}$ is a $\sigma$-companion of $f^{*} A$ for some embedding $\sigma: E(A) \rightarrow \overline{\mathbb{Q}_{\ell^{\prime}}}$, then $A^{\prime}$ is a $\sigma$-companion of $A$.

Proof. Recall that for any linear operator $F$ on a finite-dimensional $(\mathbb{Z} / 2 \mathbb{Z})$-graded vector space and any $N, \operatorname{tr}(F)$ can be recovered from the numbers $\operatorname{tr}\left(F^{n}\right), n \geq N$ linearly with coefficients in the field generated by the latter ([Z1, Section 1], [I, Lemma 8.1, Remark $8.2(3)])$. Thus it suffices to show that for any $y \in Y\left(\mathbb{F}_{q^{m}}\right)$, there exists $N \geq 1$ such that for every $n \geq N$, the image of $y$ in $Y\left(\mathbb{F}_{q^{n m}}\right)$ lifts to $X$. This follows from the lemma below.

Lemma 4.13. Let $X$ be a geometrically irreducible stack over $\mathbb{F}_{q}$. Then there exists an integer $N \geq 1$ such that $X$ admits an $\mathbb{F}_{q^{n}}$-point for every $n \geq N$.

Proof. Let $d$ be the dimension of $X$. Consider $H_{c}^{i}=H_{c}^{i}\left(X \otimes_{\mathbb{F}_{q}} \overline{\mathbb{F}_{q}}, \overline{\mathbb{Q}_{\ell}}\right)$. Then $H_{c}^{2 d} \simeq$ $\overline{\mathbb{Q}_{\ell}}(-d)$, and for $j>0, H_{c}^{2 d+j}=0$ and $H_{c}^{2 d-j}$ has weights $\leq 2 d-\frac{j}{2}$ [S1, Theorem 1.4]. Let $\iota: \overline{\mathbb{Q}_{\ell}} \rightarrow \mathbb{C}$ be an embedding. By [S1, Theorem 4.2], $M_{n}=\sum_{\alpha}|\iota \alpha|^{n}<\infty$, 
where $\alpha$ runs through the multiset of eigenvalues of Frob $q$ acting on $H_{c}^{2 d-j}, j>0$. Since $M_{n} \leq q^{(n-1)\left(d-\frac{1}{4}\right)} M_{1}$, we have $M_{n}<q^{d n}$ for $n \gg 0$. By the trace formula, we then have

$$
\sum_{x \in\left|X\left(\mathbb{F}_{q^{n}}\right)\right|} \frac{1}{\# \operatorname{Aut}(x)}=q^{d n}+\sum_{\alpha}\left( \pm \alpha^{n}\right)>0 .
$$

Here $\left|X\left(\mathbb{F}_{q^{n}}\right)\right|$ denotes the set of isomorphism classes of the groupoid $X\left(\mathbb{F}_{q^{n}}\right)$.

Finally, we deduce the existence of perverse companions and companions in Grothendieck groups.

Theorem 4.14. Let $X$ be a stack of separated diagonal. Let $\mathcal{P}$ be a perverse $W i l \overline{\mathbb{Q}_{\ell}}$-sheaf on $X$. Then, for every embedding $\sigma: E(\mathcal{P}) \rightarrow \overline{\mathbb{Q}_{\ell^{\prime}}}, \mathcal{P}$ admits a perverse $\sigma$-companion $\mathcal{P}^{\prime}$, unique up to semisimplification. Moreover, if $E(\mathcal{P})$ is a number field, then there exists a finite extension $E$ of $E(\mathcal{P})$ such that for every finite place $\lambda^{\prime}$ of $E$ not dividing $q, \mathcal{P}$ admits a perverse $\sigma_{\lambda^{\prime}}$-companion. Here $\sigma_{\lambda^{\prime}}: E(\mathcal{P}) \rightarrow E \rightarrow E_{\lambda^{\prime}}$, and $E_{\lambda^{\prime}}$ denotes the completion of $E$ at $\lambda^{\prime}$.

Proof. The uniqueness up to semisimplification follows from Chebotarev's density theorem (Proposition 4.6). For the existence of companion, we extend $\sigma$ to an embedding $\overline{\mathbb{Q}_{\ell}} \rightarrow \overline{\mathbb{Q}_{\ell^{\prime}}}$. If $E(\mathcal{P})$ is a number field, then $E\left(\mathcal{P}_{i}\right)$ is a number field for every simple factor $\mathcal{P}_{i}$ of $\mathcal{P}$ by Proposition 4.8. Thus, for the existence of companion in both assertions of the theorem, we may assume that $\mathcal{P}$ is simple. Then $\mathcal{P}$ has the form $j_{! *}(\mathcal{F}[d])$ for $j: Y \rightarrow X$ an immersion with $Y$ smooth, $\mathcal{F}$ lisse on $Y$, and $d$ the locally constant dimension function on $Y$. The existence of companion follows from Theorem 0.2 applied to $\mathcal{F}$ and the fact that $j_{\text {!* }}$ on Grothendieck groups preserves companions (Theorem 4.11).

Corollary 4.15. Let $X$ be a stack of separated diagonal. Let $\mathcal{P}$ be a simple perverse Weil $\overline{\mathbb{Q}_{\ell}}$-sheaf on $X$. Then, for every embedding $\sigma: E(\mathcal{P}) \rightarrow \overline{\mathbb{Q}_{\ell^{\prime}}}$, there exists a unique perverse $\sigma$-companion $\mathcal{P}^{\prime}$. Moreover, $\mathcal{P}^{\prime}$ is simple.

Proof. It suffices to show the simplicity. The proof is the same as the end of the proof of Proposition 4.2. We extend $\sigma$ to an isomorphism $\overline{\mathbb{Q}_{\ell}} \stackrel{\sim}{\rightarrow} \overline{\mathbb{Q}_{\ell^{\prime}}}$. We may assume $\mathcal{P}^{\prime} \simeq \bigoplus_{i} \mathcal{P}_{i}^{\prime}$ with $\mathcal{P}_{i}^{\prime}$ simple. Then for each $i$ there exists a $\mathcal{P}_{i}$ such that $\mathcal{P}_{i}^{\prime}$ is the $\sigma$-companion of $\mathcal{P}_{i}$. It follows that $\mathcal{P} \simeq \bigoplus_{i} \mathcal{P}_{i}$, so that $\mathcal{P}_{i}=0$ for all but one $i$, and the same holds for $\mathcal{P}_{i}^{\prime}$.

Corollary 4.16. Let $X$ be a stack of separated diagonal. Let $\sigma: \overline{\mathbb{Q}_{\ell}} \stackrel{\sim}{\rightarrow} \overline{\mathbb{Q}_{\ell^{\prime}}}$ be an isomorphism. For any $A \in K\left(X, \overline{\mathbb{Q}_{\ell}}\right)$, there exists a unique $\sigma$-companion $A^{\prime}$. The map $K\left(X, \overline{\mathbb{Q}_{\ell}}\right) \rightarrow K\left(X, \overline{\mathbb{Q}_{\ell^{\prime}}}\right)$ sending $A$ to its $\sigma$-companion $A^{\prime}$ is an isomorphism. Moreover, if $E(A)$ is a number field, then there exists a finite extension $E$ of $E(A)$ such that for every finite place $\lambda^{\prime}$ of $E$ not dividing $q$, A admits a perverse $\sigma_{\lambda^{\prime}}$-companion, where $\sigma_{\lambda^{\prime}}$ is as in Theorem 4.14.

Note that if $A=\sum_{\mathcal{P}} n_{\mathcal{P}}[\mathcal{P}]$, where $\mathcal{P}$ runs through isomorphism classes of simple perverse $\overline{\mathbb{Q}_{\ell}}$-sheaves, then $A^{\prime}=\sum_{\mathcal{P}} n_{\mathcal{P}}\left[\mathcal{P}^{\prime}\right]$, where $\mathcal{P}^{\prime}$ is the perverse $\sigma$-companion of $\mathcal{P}$, is the $\sigma$-companion of $A$.

Proof. The existence of $\sigma$-companion follows from Theorem 4.14 or 0.2 and the uniqueness follows from Chebotarev's density theorem. For the second assertion, note that sending $A^{\prime}$ to its $\sigma^{-1}$-companion defines an inverse of the map. For the last assertion, note that $A=[\mathcal{P}]-[\mathcal{Q}]$ with $E(\mathcal{P})$ and $E(\mathcal{Q})$ being number fields by Proposition 4.8, so that it suffices to apply the last assertion of the theorem. 
Remark 4.17. Let $X$ be a stack of separated diagonal. The group of functions $K^{W}(X, \mathbb{C}) \subseteq$ $\mathbb{C}_{n \geq 1}\left|X\left(\mathbb{F}_{q^{n}}\right)\right|$ of the form $\iota \circ t_{X}(A)$, where $A$ belongs to $K^{W}\left(X, \overline{\mathbb{Q}_{\ell}}\right)$ and $\iota: \overline{\mathbb{Q}_{\ell}} \stackrel{\sim}{\rightarrow} \mathbb{C}$ is an isomorphism, does not depend on the choice of $\ell$ and $\iota$ by Corollary 4.16. Similarly, the subgroups $K_{\text {mot }}(X, \overline{\mathbb{Q}}) \subseteq K_{\text {alg }}^{W}(X, \overline{\mathbb{Q}}) \subseteq \overline{\mathbb{Q}}^{\amalg_{n \geq 1}\left|X\left(\mathbb{F}_{q^{n}}\right)\right|}$, inverse images via an embedding $i: \overline{\mathbb{Q}} \rightarrow \overline{\mathbb{Q}_{\ell}}$ of the corresponding subgroups of $t_{X}\left(K^{W}\left(X, \overline{\mathbb{Q}_{\ell}}\right)\right)$, do not depend on the choice of $\ell$ and $i$ (cf. [D4, Corollary 1.6]). We have

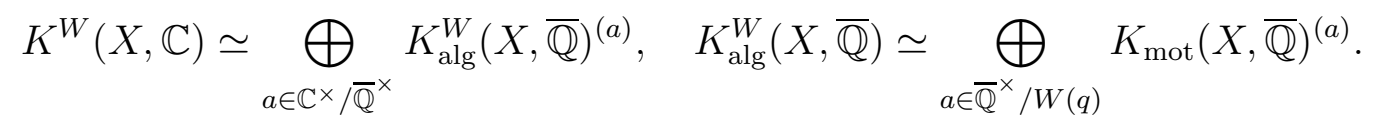

Remark 4.18. The support of a simple perverse Weil $\overline{\mathbb{Q}_{\ell}}$-sheaf $\mathcal{P}$ on a stack $X$ equals the maximal reduced closed substack $Y$ of $X$ such that $\operatorname{tr}\left(\operatorname{Frob}_{x}, \mathcal{P}_{\bar{x}}\right)=0$ for all $n \geq 1$ and all $x \in(X-Y)\left(\mathbb{F}_{q^{n}}\right)$ by Proposition 4.6. Assume that $X$ has separated diagonal. Then the perverse $\sigma$-companion $\mathcal{P}^{\prime}$ of $\mathcal{P}$ has the same support as $\mathcal{P}$. Sun [S3] defines the open support of $\mathcal{P}$ to be the maximal smooth Zariski open of $Y$ on which $\mathcal{P}$ is the shift of a lisse Weil $\overline{\mathbb{Q}_{\ell}}$-sheaf. As he observed, $\mathcal{P}$ and $\mathcal{P}^{\prime}$ have the same open support by Theorem 0.2 ,

\section{Appendix: Structure of pure perverse sheaves}

The goal of this appendix is to prove the geometric semisimplicity of pure perverse sheaves (Theorem 5.1).

Let $\iota: \overline{\mathbb{Q}_{\ell}} \rightarrow \mathbb{C}$ be an embedding. Let $X$ be a stack. Let $w \in \mathbb{R}$ and let $K \in D\left(X, \overline{\mathbb{Q}_{\ell}}\right)$. We say that $K$ has $\iota$-weights $\leq w$ if the $i$-th cohomology sheaf $\mathcal{H}^{i} K$ of $K$ has punctual $\iota$-weights $\leq w+i$ for all $i$, and $K$ has $\iota$-weights $\geq w$ if $D K$ has $\iota$-weights $\leq-w$. We say that $K$ is $\iota$-pure of weight $w$ if it has $\iota$-weights $\leq w$ and $\geq w$.

Theorem 5.1. Let $X$ be a stack and let $\mathcal{P}$ be an ı-pure perverse Weil $\overline{\mathbb{Q}_{\ell}}$-sheaf on $X$. Then the pullback of $\mathcal{P}$ to $X \otimes_{\mathbb{F}_{q}} \overline{\mathbb{F}_{q}}$ is semisimple.

The case of affine stabilizers is a theorem of Sun [S2, Theorem 3.11], extending the case of schemes [BBD, Théorème 5.3.8]. Note that the decomposition theorem of pure complexes [S2, Theorem 3.12] does not extend to general stacks, as shown in [S2, Section 1].

As in the case of schemes [BBD, Proposition 5.3.9], Theorem [5.1 has the following consequence on the structure of pure perverse sheaves. As before we let $\mathcal{E}_{n}$ denote the $\overline{\mathbb{Q}_{\ell}}$-sheaf on $\operatorname{Spec}\left(\mathbb{F}_{q}\right)$ of stalk $\overline{\mathbb{Q}}_{\ell}^{n}$ on which Frob ${ }_{q}$ acts unipotently with one Jordan block.

Corollary 5.2. Let $X$ be a stack. The indecomposable ı-pure perverse Weil $\overline{\mathbb{Q}_{\ell}}$-sheaves on $X$ are of the form $\mathcal{P} \otimes \pi_{X}^{*} \mathcal{E}_{n}$ with $\mathcal{P}$ simple, where $\pi_{X}: X \rightarrow \operatorname{Spec}\left(\mathbb{F}_{q}\right)$. Moreover, for every simple perverse Weil $\overline{\mathbb{Q}}_{\ell}$-sheaf $\mathcal{P}$, there exists a unique $m \geq 1$ such that $\mathcal{P} \simeq p_{*} \mathcal{Q}$, where $p: X \otimes_{\mathbb{F}_{q}} \mathbb{F}_{q^{m}} \rightarrow X$ is the projection, $\mathcal{Q}$ is geometrically simple (i.e. the pullback of $\mathcal{Q}$ to $X \otimes_{\mathbb{F}_{q} m} \overline{\mathbb{F}_{q}}$ is simple) and not isomorphic to any of its conjugates under $\operatorname{Gal}\left(\mathbb{F}_{q^{m}} / \mathbb{F}_{q}\right)$.

The first assertion of the corollary still holds with $\overline{\mathbb{Q}_{\ell}}$ replaced by a finite (or algebraic) extension of $\mathbb{Q}_{\ell}$.

The key to the proof of Theorem 5.1 is a weight estimate. 
Proposition 5.3. Let $X$ be a stack and let $\pi: X \rightarrow \operatorname{Spec}\left(\mathbb{F}_{q}\right)$ be the projection. Let $K \in D^{\geq 0}\left(X, \overline{\mathbb{Q}_{\ell}}\right)$ be a complex of $\iota$-weights $\geq w$ and vanishing $i$-th cohomology for $i<0$. Then for all $i \geq 0, R^{i} \pi_{*} K$ has $\iota$-weights $\geq w+\left\lceil\frac{i}{2}\right\rceil$. Moreover $H^{i}\left(X \otimes_{\mathbb{F}_{q}} \overline{\mathbb{F}_{q}}, K\right)^{\mathrm{Gal}\left(\overline{\mathbb{F}_{q}} / \mathbb{F}_{q}\right)}=0$ for $i>0$ if $w \geq 0$, and $R \Gamma(X, K)=0$ if $w>0$.

The estimate is optimal. Indeed, for $X=B A$, where $A$ is an Abelian variety, and $a$ of weight $1, R^{i} \pi_{*}\left(\overline{\mathbb{Q}_{\ell}} \oplus \overline{\mathbb{Q}}_{\ell}^{(a)}[-1]\right)$ is pure of weight $\left\lceil\frac{i}{2}\right\rceil$. Unlike the case of schemes or stacks with affine stabilizers, $R^{i} \pi_{*} K$ is not of $\iota$-weights $\geq w+i$ in general.

Proof. The second assertion follows from the first one and the short exact sequence

$$
0 \rightarrow H^{i-1}\left(X \otimes_{\mathbb{F}_{q}} \overline{\mathbb{F}_{q}}, K\right)_{\operatorname{Gal}\left(\overline{\mathbb{F}_{q}} / \mathbb{F}_{q}\right)} \rightarrow H^{i}(X, K) \rightarrow H^{i}\left(X \otimes_{\mathbb{F}_{q}} \overline{\mathbb{F}_{q}}, K\right)^{\operatorname{Gal}\left(\overline{\mathbb{F}_{q}} / \mathbb{F}_{q}\right)} \rightarrow 0 .
$$

Note that for any stratification of $X$ into locally closed substacks $\left(j_{\alpha}: X_{\alpha} \rightarrow X\right)_{\alpha}$ such that the closure of every stratum is a union of strata, $K$ is a successive extension of $R j_{\alpha *} R j_{\alpha}^{!} K$, with $R j_{\alpha}^{!} K \in D^{\geq} 0$ of $\iota$-weights $\geq w$. Thus we may assume that $X$ is smooth of dimension $d$ and $K$ has lisse cohomology sheaves. We may further assume $K=\mathcal{F}[-n]$, with $\mathcal{F}$ lisse of $\iota$-weights $\geq w+n$ and $n \geq 0$. Then the $\iota$-weights of $\left(R^{i} \pi_{*} K\right)^{\vee} \simeq\left(R^{2 d+n-i} \pi_{!} \mathcal{F}^{\vee}\right)(d)$ are at most

$$
d+\frac{2 d+n-i}{2}-(w+n)-2 d=-w-\frac{i+n}{2} \leq-w-\frac{i}{2}
$$

by [S1, Theorem 1.4]. We conclude by the fact that the $\iota$-weights are in $w+\mathbb{Z}$.

Corollary 5.4. Let $X$ be a stack and let $\mathcal{P}$ and $\mathcal{Q}$ be perverse $\overline{\mathbb{Q}}$-sheaves on $X$, with $\mathcal{P}$ of $\iota$-weights $\leq w$, and $\mathcal{Q}$ of $\iota$-weights $\geq w$. Then for $i>0, \operatorname{Hom}^{i}\left(\mathcal{P}_{\overline{\mathbb{F}_{q}}}, \mathcal{Q}_{\overline{\mathbb{F}_{q}}}\right) \operatorname{Gal}\left(\overline{\mathbb{F}_{q}} / \mathbb{F}_{q}\right)=0$, so that the canonical map $\operatorname{Hom}^{i}(\mathcal{P}, \mathcal{Q}) \rightarrow \operatorname{Hom}^{i}\left(\mathcal{P}_{\overline{\mathbb{F}_{q}}}, \mathcal{Q}_{\overline{\mathbb{F}_{q}}}\right)$ is zero. Moreover, if $\mathcal{Q}$ has $\iota$-weights $>$, then $R \operatorname{Hom}(\mathcal{P}, \mathcal{Q})=0$.

For perverse Weil $\overline{\mathbb{Q}_{\ell}}$-sheaves and $i=1$, the first assertion holds with Hom ${ }^{1}$ replaced by $\operatorname{Ext}^{1}$ and $\operatorname{Gal}\left(\overline{\mathbb{F}_{q}} / \mathbb{F}_{q}\right)$ replaced by $W\left(\overline{\mathbb{F}_{q}} / \mathbb{F}_{q}\right)$.

Proof. We apply the proposition to $K=R \mathcal{H} \operatorname{om}(\mathcal{P}, \mathcal{Q}) \in D^{\geq 0}\left(X, \overline{\mathbb{Q}_{\ell}}\right)$, which has $\iota$-weights $\geq 0$. If $\mathcal{Q}$ has $\iota$-weights $>w$, then $K$ has $\iota$-weights $>0$.

The proof of Theorem 5.1 is then identical to the proof of [BBD, Théorème 5.3.8], with [BBD, Proposition 5.1.15] replaced by Corollary 5.4.

\section{References}

[EGAI] A. Grothendieck, Éléments de géométrie algébrique. I. Le langage des schémas, Inst. Hautes Études Sci. Publ. Math. 4 (1960), 5-228. Rédigés avec la collaboration de J. Dieudonné. MR0217083 $\uparrow 12$

[SGA1] Revêtements étales et groupe fondamental (SGA 1), Documents Mathématiques (Paris) [Mathematical Documents (Paris)], vol. 3, Société Mathématique de France, Paris, 2003 (French). Séminaire de géométrie algébrique du Bois Marie 1960-61. [Algebraic Geometry Seminar of Bois Marie 1960-61]; Directed by A. Grothendieck; With two papers by M. Raynaud; Updated and annotated reprint of the 1971 original [Lecture Notes in Math., 224, Springer, Berlin; MR0354651 (50 \#7129)]. MR2017446 个6

[SGA4] Théorie des topos et cohomologie étale des schémas, Lecture Notes in Mathematics, Vol. 269, 270, 305, Springer-Verlag, Berlin, 1972-1973. Séminaire de Géométrie Algébrique du Bois-Marie 1963-1964 (SGA 4). Dirigé par M. Artin, A. Grothendieck, et J. L. Verdier. Avec la collaboration de N. Bourbaki, P. Deligne et B. Saint-Donat. MR0354652 (50 \#7130), MR0354653 (50 \#7131), MR0354654 (50 \#7132) T5 
[SGA7II] P. Deligne and N. Katz, Groupes de monodromie en géométrie algébrique. II, Lecture Notes in Mathematics, Vol. 340, Springer-Verlag, Berlin-New York, 1973 (French). Séminaire de Géométrie Algébrique du Bois-Marie 1967-1969 (SGA 7 II). MR0354657 110

[A] T. Abe, Langlands correspondence for isocrystals and existence of crystalline companion for curves, J. Amer. Math. Soc., DOI 10.1090/jams/898. 112

[B] K. A. Behrend, Derived l-adic categories for algebraic stacks, Mem. Amer. Math. Soc. 163

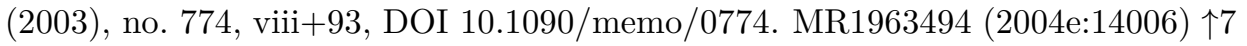

[BBD] A. A. Beĭlinson, J. Bernstein, and P. Deligne, Faisceaux pervers, Analysis and topology on singular spaces, I (Luminy, 1981), Astérisque, vol. 100, Soc. Math. France, Paris, 1982, pp. 5171 (French). MR751966 (86g:32015) 14, 13, 18, 23, 24,

[BR] J. Bénabou and J. Roubaud, Monades et descente, C. R. Acad. Sci. Paris Sér. A-B 270 (1970), A96-A98 (French). MR0255631 个5

[B1] N. Bourbaki, Éléments de mathématique. Algèbre. Chapitres 4 à 7, Springer, 2007 (French).

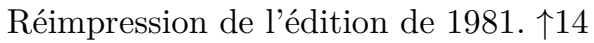

[B2] Éléments de mathématique. Algèbre. Chapitre 8. Modules et anneaux semi-simples, Springer, Berlin, 2012 (French). Second revised edition of the 1958 edition [MR0098114]. MR3027127 个18

[C] C. Chin, Independence of $\ell$ in Lafforgue's theorem, Adv. Math. 180 (2003), no. 1, 64-86, DOI 10.1016/S0001-8708(02)00082-8. MR2019215 (2005b:14041) 怆

[CR] C. W. Curtis and I. Reiner, Representation theory of finite groups and associative algebras, Pure and Applied Mathematics, Vol. XI, Interscience Publishers, a division of John Wiley \& Sons, New York-London, 1962. MR0144979 119

[D1] P. Deligne, Les constantes des équations fonctionnelles des fonctions L, Modular functions of one variable, II (Proc. Internat. Summer School, Univ. Antwerp, Antwerp, 1972), Springer, Berlin, 1973, pp. 501-597. Lecture Notes in Math., Vol. 349 (French). MR0349635 115

[D2] — La conjecture de Weil. II, Inst. Hautes Études Sci. Publ. Math. 52 (1980), 137-252 (French). MR601520 (83c:14017) 1, 2, 6, 7, 8, 13, 16,

[D3] _ Finitude de l'extension de $\mathbb{Q}$ engendrée par des traces de Frobenius, en caractéristique finie, Mosc. Math. J. 12 (2012), no. 3, 497-514, 668 (French, with French and Russian summaries). MR3024820 个2, 6, 9, 10, 14,

[D4] V. Drinfeld, On a conjecture of Deligne, Mosc. Math. J. 12 (2012), no. 3, 515-542, 668 (English,

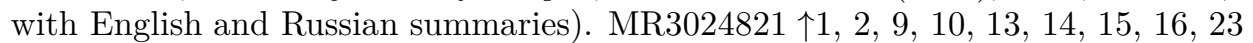

[DK] V. Drinfeld and K. Kedlaya, Slopes of indecomposable F-isocrystals (2018). arXiv:1604.00660v9, preprint. 12, 11, 12

[EK] H. Esnault and M. Kerz, A finiteness theorem for Galois representations of function fields over

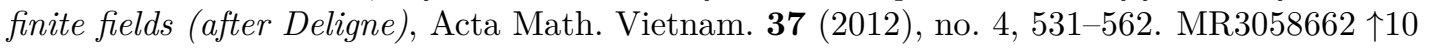

[F] K. Fujiwara, Independence of l for intersection cohomology (after Gabber), Algebraic geometry 2000, Azumino (Hotaka), Adv. Stud. Pure Math., vol. 36, Math. Soc. Japan, Tokyo, 2002, pp. 145-151. MR1971515 (2004c:14038) 个3, 21]

[G] J. Giraud, Méthode de la descente, Bull. Soc. Math. France Mém. 2 (1964), viii+150 (French). MR0190142 14, 5]

[I] L. Illusie, Miscellany on traces in $\ell$-adic cohomology: a survey, Jpn. J. Math. 1 (2006), no. 1, 107-136, DOI 10.1007/s11537-006-0504-3. MR2261063 个21

[IZ] L. Illusie and W. Zheng, Quotient stacks and equivariant étale cohomology algebras: Quillen's theory revisited, J. Algebraic Geom. 25 (2016), no. 2, 289-400. MR3466353 㥜, 7

[K] D. Knutson, Algebraic spaces, Lecture Notes in Mathematics, Vol. 203, Springer-Verlag, BerlinNew York, 1971. MR0302647 个10 
[L1] L. Lafforgue, Chtoucas de Drinfeld et correspondance de Langlands, Invent. Math. 147 (2002), no. 1, 1-241, DOI 10.1007/s002220100174 (French, with English and French summaries). MR1875184 (2002m:11039) 㤷 10, 11

[L2] V. Lafforgue, Estimées pour les valuations p-adiques des valeurs propes des opérateurs de Hecke, Bull. Soc. Math. France 139 (2011), no. 4, 455-477 (French, with English and French summaries). MR2869300 110, 11]

[L3] G. Laumon, Transformation de Fourier, constantes d'équations fonctionnelles et conjecture de Weil, Inst. Hautes Études Sci. Publ. Math. 65 (1987), 131-210 (French). MR908218 (88g:14019) 118

[LMB] G. Laumon and L. Moret-Bailly, Champs algébriques, Ergebnisse der Mathematik und ihrer Grenzgebiete. 3. Folge. A Series of Modern Surveys in Mathematics, vol. 39, Springer-Verlag, Berlin, 2000. MR1771927 (2001f:14006) 17, 8, 15, 21

[M] D. Mumford, Abelian varieties, Tata Institute of Fundamental Research Studies in Mathematics, vol. 5, Published for the Tata Institute of Fundamental Research, Bombay; by Hindustan Book Agency, New Delhi, 2008. With appendices by C. P. Ramanujam and Yuri Manin; Corrected reprint of the second (1974) edition. MR2514037 (2010e:14040) 110

[N] B. Noohi, Fundamental groups of algebraic stacks, J. Inst. Math. Jussieu 3 (2004), no. 1, 69-103, DOI 10.1017/S1474748004000039. MR2036598 佔

[R] D. Rydh, Submersions and effective descent of étale morphisms, Bull. Soc. Math. France 138 (2010), no. 2, 181-230 (English, with English and French summaries). MR2679038 10

[SP] The Stacks Project Authors, Stacks Project. http://stacks.math.columbia.edu. 町 7

[S1] S. Sun, L-series of Artin stacks over finite fields, Algebra Number Theory 6 (2012), no. 1, 47-122, DOI 10.2140/ant.2012.6.47. MR2950161 112, 13, 17, 21, 24.

[S2] _ Decomposition theorem for perverse sheaves on Artin stacks over finite fields, Duke Math. J. 161 (2012), no. 12, 2297-2310, DOI 10.1215/00127094-1723657. MR2972459 惔 12 , 13. 23

[S3] _ Independence of $\ell$ for the supports in the decomposition theorem (2015). arXiv: $1512.01447 \mathrm{v} 1$, preprint. $\uparrow 23$

[SZ] S. Sun and W. Zheng, Parity and symmetry in intersection and ordinary cohomology, Algebra Number Theory 10 (2016), no. 2, 235-307, DOI 10.2140/ant.2016.10.235. MR3477743 㥀 18, 19, 20,

[V] V. Voevodsky, Homology of schemes, Selecta Math. (N.S.) 2 (1996), no. 1, 111-153, DOI 10.1007/BF01587941. MR1403354 佔

[Z1] W. Zheng, Théorème de Gabber d'indépendance de l (2005) (French). Mémoire de Master deuxième année, arXiv:1608.06191, 120, 21

[Z2] _ Sur la cohomologie des faisceaux l-adiques entiers sur les corps locaux, Bull. Soc. Math. France 136 (2008), no. 3, 465-503 (French, with English and French summaries). MR2415350

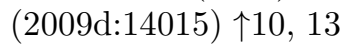

[Z3] _ Sur l'indépendance de l en cohomologie l-adique sur les corps locaux, Ann. Sci. Éc. Norm. Supér. (4) 42 (2009), no. 2, 291-334 (French, with English and French summaries). MR2518080 (2010i:14032) 13, 15, 17, 21, 